\title{
Lipidomic Approaches towards Deciphering Glycolipids from Microalgae as a Reservoir of Bioactive Lipids
}

\author{
Elisabete da Costa ${ }^{1}$, Joana Silva ${ }^{2}$, Sofia Hoffman Mendonça ${ }^{2}$, Maria Helena Abreu ${ }^{3}$ \\ and Maria Rosário Domingues ${ }^{1, *}$ \\ 1 Centro de Espectrometria de Massa, Departamento de Química \& QOPNA, Universidade de Aveiro, \\ Campus Universitário de Santiago, 3810-193 Aveiro, Portugal; elisabetecosta@ua.pt \\ 2 Allmicroalgae-Natural Products S.A., Avenida das Forças Armadas, 125, $7^{\circ}$ piso, 1600-079 Lisboa, Portugal; \\ jglaranjeira2@gmail.com (J.S.); sofia.mendonca@allmicroalgae.com (S.H.M.) \\ 3 ALGAplus-Produção e Comercialização de Algas e Derivados, Lda., 3830-196 Ílhavo, Portugal; \\ htabreu@algaplus.pt \\ * Correspondence: mrd@ua.pt; Tel.:+351-234-370-698
}

Academic Editor: Andrew Desbois

Received: 19 February 2016; Accepted: 12 May 2016; Published: 19 May 2016

\begin{abstract}
In recent years, noteworthy research has been performed around lipids from microalgae. Among lipids, glycolipids (GLs) are quite abundant in microalgae and are considered an important source of fatty acids (FAs). GLs are rich in 16- and 18-carbon saturated and unsaturated fatty acids and often contain polyunsaturated fatty acids (PUFAs) like $n$-3 $\alpha$-linolenic (ALA 18:3), eicosapentaenoic (EPA, 20:5) and docosahexaenoic (DHA, 22:6). GLs comprise three major classes: monogalactosyldiacyl glycerolipids (MGDGs), digalactosyl diacylglycerolipids (DGDGs) and sulfoquinovosyl diacylglycerolipids (SQDGs), whose composition in FA directly depends on the growth conditions. Some of these lipids are high value-added compounds with antitumoral, antimicrobial and anti-inflammatory activities and also with important nutritional significance. To fully explore GLs' bioactive properties it is necessary to fully characterize their structure and to understand the relation between the structure and their biological properties, which can be addressed using modern mass spectrometry (MS)-based lipidomic approaches. This review will focus on the up-to-date FA composition of GLs identified by MS-based lipidomics and their potential as phytochemicals.
\end{abstract}

Keywords: lipidomic; glycolipids; microalgae; fatty acids; mass spectrometry; bioprospection

\section{Introduction}

Microalgae are a group of unicellular photosynthetic microscopic organisms classified as prokaryotic (bacteria, Kingdom Monera) and eukaryotic (Kingdom Protista) further distributed into several divisions, depending on their pigmentation and physiology [1,2]. Among the most common divisions there are the prokaryotic Cyanophyceae known as cyanobacteria, the eukaryotic Chlorophyceae (green algae) and the Bacillariophyceae (Heterokont) [1-3]. Microalgae are widely found in aquatic and terrestrial habitats mostly due to their particular versatility in adjusting to distinct growth conditions [4]. Due to this capacity, microalgae are currently cultivated on a large scale, both in artificial or natural ponds and in photoreactors (PBR), representing an important group of organisms for biotechnological exploitation with an important impact in the food and pharmaceutical industries and public health [5-7]. The microalgae-based product market has reached a size of about 8000 ton/year of dry matter, generating an annual turnover of U.S. $\$ 4 \times 10^{8} /$ year [5]. The biomass produced by several species like Dunaliella, Arthrospira (Spirulina) and Chlorella is already being marketed in various forms 
designed for human nutrition, either incorporated into foods and beverages or as a healthy nutritional supplement, rich in carbohydrates, proteins, vitamins, minerals, pigments and lipids [6-8].

Nowadays, due to the high content of lipids, there has been increasing attention in the use of microalgae as a source of lipids with various commercial applications, for example, as supplementary food or in the chemical, pharmaceutical and cosmetic industries [8,9]. Lipids of some microalgae species are enriched in valuable polyunsaturated fatty acids (PUFAs) that are mainly esterified to other lipids. Such lipids can be neutral/non-polar lipids like mono, di- and tri-acylglycerides, or polar lipids including phospholipids and glycolipids [10-12]. Glycolipids (GLs) represent a less studied class of lipids that captured the growing interest of researchers. They are located in the membrane of chloroplasts and thylakoids, and are important signal and regulatory molecules [11-13]. The most abundant glycolipids found in microalgae are monogalactosyl diacylglycerols (MGDGs), digalactosyl diacylglycerols (DGDGs) and sulfoquinovosyl diacylglycerols (SQDGs), which are rich in PUFAs, namely linoleic (LA, 18:2n-6), arachidonic (ARA, 20:4n-6), $\alpha$-linolenic (ALA, 18:3n-3), eicosapentaenoic (EPA, 20:5n-3) and docosahexaenoic (DHA, 22:6n-3) fatty acids [11,14,15]. GLs from microalgae are considered an important nutritional source of $n-3$ fatty acids with beneficial health effects to humans, contributing to the balance between $n-3$ and $n-6$ FAs (fatty acids). In addition, some GLs extracted from microalgae and macroalgae possess biological activities such as antifungal, antiviral and antitumoral properties [16-21]. They also present health benefits as anti-inflammatory and with antimicrobial properties [17]. The growing resistance of pathogenic bacteria against commercially available antimicrobial drugs turns the search for new microbial substances into one of increasing importance. The biological activity of GLs is dependent on the sugar moiety and acyl chains, however the specific relation of structure-activity remains unknown [22].

The detailed characterization of GLs structures and profile from microalgae is not an easy task due to the high number of different GLs molecular species present in the full lipidome. Additionally, the changes in the content and composition of GLs, namely the composition in fatty acids that may occur within the same microalgae species, depends on the growth and environmental conditions such as light, salinity, temperature, contaminants parameters and nutrient availability, increasing the large chemical diversity and complexity of the structures [10,15,23].

Until now, few studies focusing on the glycolipidome of microalgae have been performed due to the complexity of the full lipidome [14,15,24-28]. Considering the recognized biological role of some glycolipids, more efforts are needed to identify and evaluate the structural features of GLs and the correspondent signature of each microalgae. To overcome this challenge, MS-based approaches represent a new emerging technology that will provide new insights which are capable of affording a better understanding of the mechanisms involved in GLs activities. This work presents a succinct review of the studies, including MS-based approaches, towards deciphering the structure of glycolipids isolated from microalgae and their potential bioactive properties.

\section{The Structure of Glycolipids}

Glycolipids in microalgae are glyceroglycolipids that have a glycerol backbone which anchors one or two acyl chains esterified $\left(R_{1}\right.$ and $\left.R_{2}\right)$ at positions $s n-1$ and $s n-2$, and a sugar moiety linked at position $s n-3$ in a $\beta$-anomeric linkage (Figure 1) [29]. Depending on the composition of the glycosidic head group, the GLs can be classified as monogalactosyl diacylglycerol (MGDG), digalactosyl diacylglycerol (DGDG) and sulfoquinovosyl diacylglycerol (SQDG) [10,13]. Each GLs class can accommodate several molecular species depending on the fatty acids (FA) that are linked to $s n-1$ and $s n-2$ positions of the glycerol backbone. FAs with different chain lengths and degrees of unsaturation can be distributed in $s n-1$ and $s n-2$ positions of glycerol $[29,30]$. The properties of GLs directly depend on the sugar moiety and on the length, degree of unsaturation and regiospecificity of the two acyl chains in the $s n-1$ and $s n-2$ positions of glycerol [22].

The predominant GLs in microalgae are the neutral and uncharged galactosylglycerides, MGDGs and DGDGs. MGDG contains one galactose $\beta$-anomeric linked to the $s n-3$ position of 
glycerol backbone corresponding to 1,2-diacyl-3-O-( $\beta$-D-galactopyranosyl)-sn-glycerol. DGDG is characterized by a terminal $\alpha$-galactose moiety $(1 \rightarrow 6)$ linked to the inner $\beta$-galactose residue, also named 1,2-diacyl-3-O-( $\alpha$-galactosyl-(1,6)-O- $\beta$-D-galactopyranosyl-sn-glycerol. MGDG and DGDG molecular species are characterized by their high content of PUFA, mostly 16- and 18-carbon $n-3$ fatty acids, and often contain very long chain polyunsaturated acids, with more than 20 carbon atoms and more than three double bonds such as eicosapentaenoic acid (EPA) [29,30]. MGDG tends to adapt a conical shape if the fatty acids are highly unsaturated (non-bilayer-forming lipid). Meanwhile, MGDG containing saturated fatty acids preferably adapt to a cylindrical form, while DGDG has a more cylindrical shape and forms lipid bilayers (Figure 1) [31]. Under nutrition starvation and stress conditions, DGDG is exported to various extraplastidial membranes, substituting for phosphoglycerolipids [32-34]. Thus, MGDG and DGDG play important roles in the structural stabilization and function of membranes [35-37], and are fundamental in the trafficking of lipids between subcellular compartments [35].
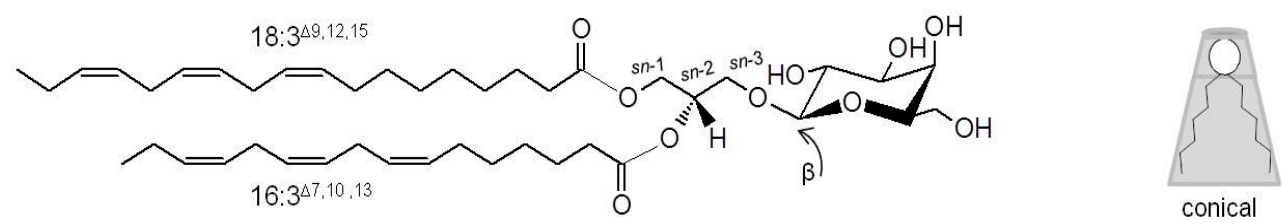

Monogalactosyl diacylglycerol (MGDG)
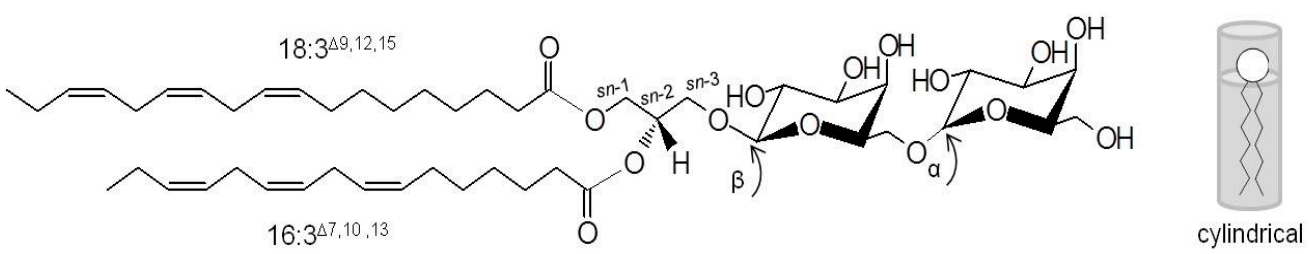

Digalactosyl diacylglycerol (DGDG)

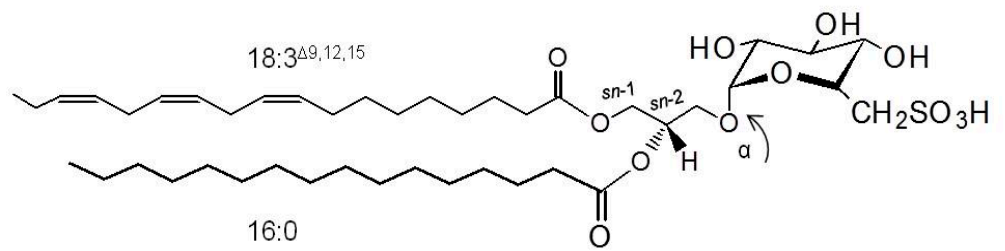

Sulfoquinovosyl diacylglycerol (SQDG)

Figure 1. Main glycerolipid classes conserved in photosynthetic membranes of algae: MGDG, monogalactosyl diacylglycerol; DGDG, diagalactosyl diacylglycerol; SQDG, sulfoquinovosyl diacylglycerol; $\mathrm{R}_{1}$ and $\mathrm{R}_{2}$ represent fatty acyl chains. In the membrane structure of chloroplasts, MDGD tends to adapt a conical shape; DGDG and SQDG tend to adapt cylindrical shapes.

SQDG is a negatively charged GL composed of a monoglycosyl diacylglycerol with a sulfonic acid linked in the position 6 of the monosaccharide moiety (1,2-diacyl-3-O-(6-sulfo-6-deoxy- $\alpha$-D-glucosyl)-sn-glycerol [19,20,38,39]. The presence of the sulfoquinovosyl moiety is responsible for the negative charge of the sulfonic residue at physiological $\mathrm{pH}$ [38]. SQDG molecular species have a high content of hexadecanoic acid (16:0), octadecadienoic acid (18:2), eicosatetraenoic acid (20:4) and eicosapentaenoic acid (20:5) [19,40]. SQDG preferably adapts a cylindrical shape suitable for packing side by side in a bilayer [41]. SQDGs play important roles in the "membrane mosaic", particularly in signaling and in the coordination between chloroplast lipids (outer envelope membrane) and cytosolic partners [41]. 
MGDG, DGDG and SQDG are major components of the plastid lipids [12,35,41]. MGDG constitutes the outer $(20 \%)$ and the inner envelope of chloroplast and thylakoid membranes $(40 \%-55 \%)$ [12]. DGDG represents about $15 \%-35 \%$ and SQDG about $2 \%-40 \%$ of total lipids in both chloroplast and thylakoid membranes [12]. The content of SQDG in microalgae is relatively high when compared with other green plants ( $2 \%-10 \%)$, like Arabidopsis thaliana [12,30,41]. Glycolipids bearing only one fatty acyl chain (lysoglycolipids) can be found in microalgae, although with low abundance, such as monoacyl monogalactolipids (MGMGs), monoacyl digalactolipids (DGMGs) and monoacyl sulfoquinovosyl lipids (SQMGs) [42]. Also glycolipids containing three galactoses (trigalactosyl diacylglycerol, TGDG) were reported in dinoflagellate glycolipidome [43]. TGDGs were previously described in the glycolipidome of the plant Arabidopsis [44]. Digalactosyl triacylglycerol (DGTG) and sulfoquinovosyl triacylglycerol, with a fatty acyl moiety esterified at the C-3 of the sugar unit, were described in the lipidome of cyanobacteria [20].

The roles and functions of GLs depend on their structure and composition, the coordination of which is directly dependent on biosynthetic pathways. Glycolipids are mainly synthesized in the chloroplast, within the envelope membranes of plastids, by the assembly of a glycosidic moiety to diacylglycerol (DAG) [29]. This biosynthesis is orchestrated by the activities of a panel of enzymes that coordinate the synthesis of each specific lipid, the trafficking of lipid intermediates and the catabolic pathways of lipids [35]. Specific enzymes control the type of sugar linked to the polar head, the type of fatty acid and its position in the glycerol backbone. The two major biosynthetic pathways of glycolipids in microalgae, such as in plants, are the chloroplastic or "prokaryotic" biosynthetic pathway, that occur exclusively in the chloroplast, and the endoplasmic or "eukaryotic" pathway, that starts in the endoplasmic reticulum (ER) and ends in the chloroplast (Figure 2) [45,46]. In the "prokaryotic" pathway, the biosynthesis of DAG is catalyzed by acyltransferase proteins in the inner-envelope membrane of chloroplasts, which transfer $\mathrm{C}_{16}$ FA to the $s n-2$ position of glycerol $[10,12]$. Galactolipids are further synthesized by two different galactosyltransferases (Figure 2), each transferring a galactosyl residue from uridine diphospho-galactose (UDP-Gal) to the $s n-3$ position of DAG, to synthesize MGDG, or to MGDG to form DGDG. The anomeric configuration is a $\beta$-glycosidic linkage in the first sugar and a $\alpha$-glycosidic linkage in the second $[27,29,36]$. SQDG is produced in the chloroplast's envelope by the assemblage of a sulfoquinovose head group from the UDP-sulfoquinovose conjugated to DAG. Thus the GLs from the "prokaryotic" pathway have typically 16:0 or 16:1 at sn-2 [36,41].

DAG may be derived from the ER, where it is formed during the glycerolipid biosynthetic pathway, followed by subsequent transfer of DAG moieties into the chloroplast to be used in the endoplasmic or "eukaryotic" pathway. The biosynthesis of DAGs with $\mathrm{C}_{18}$ FA at the sn-2 position, which is a signature for the ER origin of a DAG, while either $C_{16}(16: 0,16: 1)$ or $C_{18}$ fatty acids (18:0, 18:1, 18:2 or 18:3) can be at the $s n-1$ position [36,44-48]. These pathways depend on the export of some of the fatty acids made in the plastids to the ER to be used in the biosynthesis of structural lipids for the non-chloroplast membranes [44,49]. The "prokaryotic" vs. "eukaryotic" biosynthetic pathways determine the position of the fatty acyl chains in the glycerol backbone of glycolipids, particularly concerning the fatty acids $\mathrm{C}_{16}$ and $\mathrm{C}_{18}$, at the $s n-1$ or $s n-2$ positions. In fact, in the "prokaryotic" pathway, the glycolipids have mainly $\mathrm{C}_{18} \mathrm{FA}$ at the $s n-1$ position while the $\mathrm{C}_{16}$ are mainly at the $s n-2$, and the opposite is observed for the glycolipids synthesized by the "eukaryotic" pathways.

However, glycolipids can also include PUFAs like 20:4, 20:5 and 22:6, formed by the desaturation and elongation reactions occurring in the ER (Figure 2) and $C_{14}$ FA that can be biosynthesized via "prokaryotic" and/or "eukaryotic" routes [29,30,49-51]. In the chloroplast, glycolipids can undergo little turnover by deacylation-reacylation reactions [30].

In cyanobacteria, the biosynthesis of GLs is different and it has been described that the first glycolipid formed is the monoglucosyl diacylglycerol (MGlcD) followed by a second epimerisation step converting MGlcD into MGDG [13,29]. The glycolipids MGDG, DGDG and SQDG are the predominant GLs in cyanobacteria which preferably contain $C_{18}$ at $s n-1$ and $C_{16}$ at $s n-2$ [13]. 


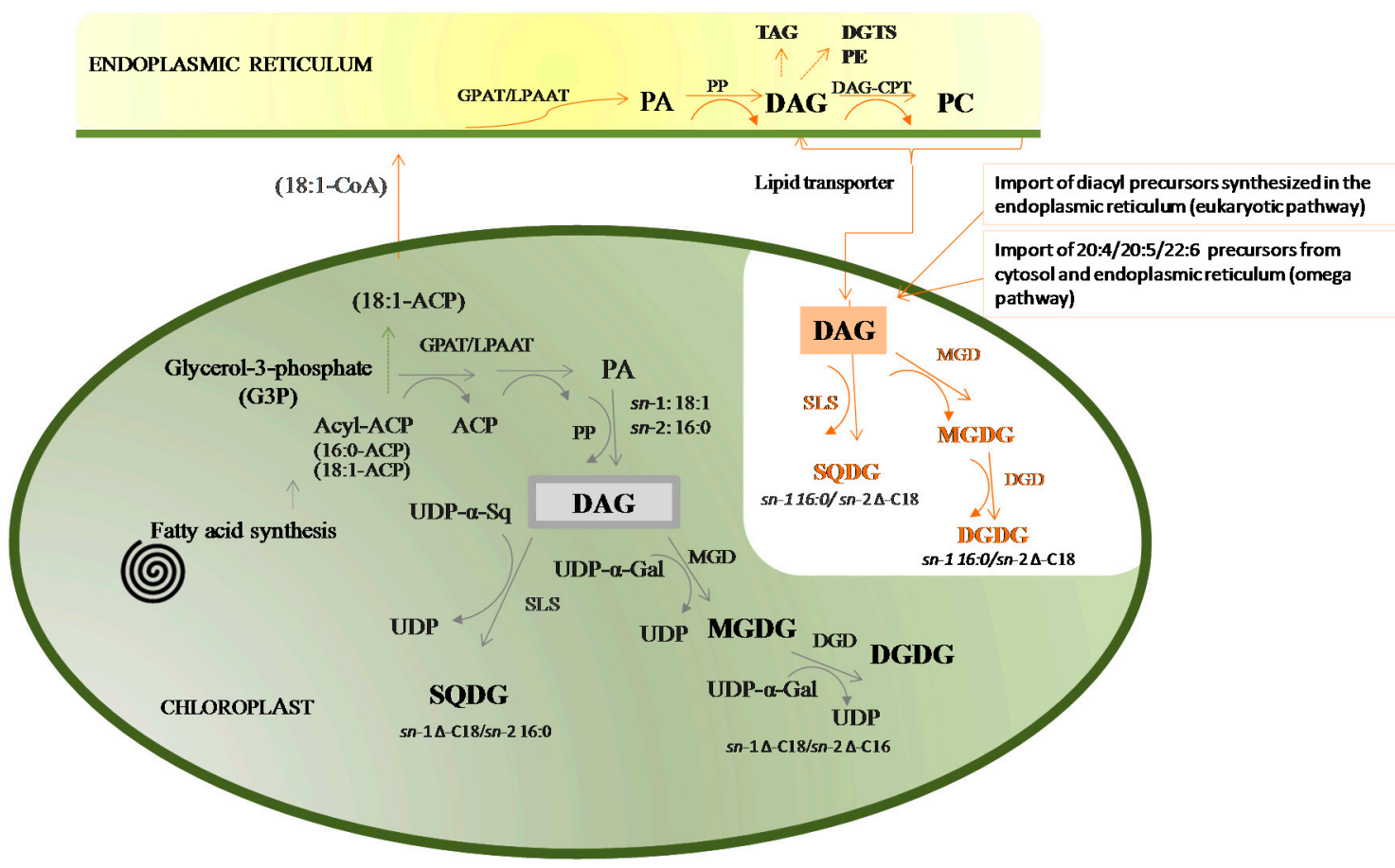

Figure 2. Simplified diagram of the main pathways involving the biosynthesis of glycerolipids in microalgae. The set of reactions occurring within the chloroplast are termed the chloroplastic or "prokaryotic" pathway and those that involve glycerolipid synthesis in the ER (endoplasmic reticulum) and subsequent transfer to the chloroplast constitute the endoplasmic or "eukaryotic" pathway. Orange arrows refer to the biosynthetic pathway of transport of ER-derived glycerolipids to chloroplasts. Enzymes involved in the biosynthesis of glycolipids are shown. ACP, acyl carrier protein; PA, phosphatidic acid; DAG, diacylglycerol; PC, phosphatidylcholine; MGD, MGDG syntases; DGD, DGDG synthases; UDP, uridine diphosphate galactose intermediate in the production of polysaccharides (-Gal galactose, -Sq sulfoquinovose); GPAT, glycerol-3-phosphatase acyltransferase; LPAAT, lysophosphatidic acid acyltransferase; PA, phosphatidic acid; PP, phosphatidate phosphatase; DAG-CPT, diacylglycerol synthetase-choline: diacylglycerol cholinephosphotransferase; SLS, sulfolipid synthase; SQDG, sulfoquinovosyl diacylglycerol; PE, phosphatidylethanolamine; DGTS, diacylglyceryl trimethylhomo-serine; TAG, triacylglycerol; $\Delta$, degree of unsaturation ranging from 1 to 4 double bonds.

The existent knowledge of the membrane lipids biosynthesis was mainly based on studies obtained from the green algae model Chlamydomonas reinhardtii [12,36] and from the plant Arabidopsis thaliana [44-48], but there is still a great deal to learn in this area. Clearly, more knowledge is needed concerning the structural details of glycolipids, the biosynthesis pathways of the distinct lineages and the distinctive roles of the membrane lipids, providing fascinating fields of research. The structural complexity of polar lipids and the knowledge of the biosynthetic pathway can be improved with the new advances on high resolution and accurate mass and tandem mass spectrometry technology [14,48,52,53].

\section{Biological Properties Associated with Glycolipids from Microalgae}

Glycolipids are a class of metabolites that recently has gathered interest because of their potential biotechnological applications. Moreover, they are considered promising phytochemicals with a wide range of biological properties such as antimicrobial, anti-microfouling, antitumor promoting and anti-inflammatory $[9,17,23,54-56]$. In addition, GLs isolated from marine algae seem to have modulatory effects on oxidative stress, on the inhibition effect on the production of NO and on oxidative stress-related diseases and cancers, carrying several beneficial health effects (Table 1) $[9,16,23,54]$. 
The anti-inflammatory effect of GLs extracts from the Porphyridium [57], Tetraselmis [58] and Nannochloropsis [59] spp. were evaluated via inhibition of lipopolysaccharide (LPS)-induced NO production in RAW264.7 cells and on the down regulation of inducible nitric oxide synthase expression. The GLs bioactive extract of Tetraselmis contained MGDG (18:3/16:4) and MGDG (18:4/16:4) species, otherwise the bioactive extract of Nannochloropsis contained four molecular species of MGDG, namely MGDG (20:5/14:0), MGDG (20:5/16:0), MGDG (20:5/16:1) and MGDG (20:5/20:5). It has been demonstrated that polar lipids extract isolated from red algae and rich in GLs had stronger anti-inflammatory activity than pure EPA isolated from the same species, suggesting that the entire polar lipids structure contributes to anti-inflammatory activity [60]. Lipid extracts from Porphyridium were found to inhibit $\mathrm{NO}$ accumulation and may be a beneficial therapeutic strategy for the treatment of NO-mediated disorders [57]. The isolated extract was found to contain sulfolipids (five molecular species) with large amounts of palmitic acid (16:0, 26.1\%), arachidonic acid (20:4, 36.8\%) and eicosapentaenoic $(20: 5,16.6 \%)$ acids and noticeable amounts of palmitoleic acid (16:1, 10.5\%).

In order to better understand the relation between the structure and anti-inflammatory activity of sulfolipids and galactolipids, purified MGDG (18:4/16:0), DGDG (18:4/16:0) and SQDG (18:3/16:0) from thermophilic blue-green alga ETS-05 were tested in two specific in vivo mouse models: croton oil-induced ear oedema and carrageenan-induced paw oedema [61]. Both MGDG and DGDG showed biological activity. SQDG showed less anti-inflammatory effects. MGDG with a high content of saturated FA showed lower activity and SQDG seems to be the less potent GL [61].

Glycolipids are also considered important antitumor agents. SQDGs afford inhibitory effects on tumor cell growth $[10,18,21]$ and are a potent inhibitor of deoxyribonucleic acid (DNA) polymerase that can lead to the death of tumor cells, especially under conditions of active proliferation [62,63]. A lipid fraction rich in SQDG isolated from Porphyridium cruentum showed inhibitory effects on human colon (DLD-1), breast (MCF-7), prostate adenocarcinoma (PC-3) and malignant melanoma (M4-Beu) cell lines [57]. This bioactive fraction contained SQDGs with 16:0, 16:1, 20:4 and 20:5 fatty acids. MGDG and DGDG molecular species isolated from the green alga Chlorella vulgaris expressed antitumor activities by the inhibition of EBV-EA activation using EBV genome-carrying human lymphoblastoid cells (Raji cells) [21]. In particular, MGDG (16:2/16:2) and MGDG (16:3/16:2) have shown a higher inhibitory effect.

Antiviral properties of the glycolipids were demonstrated on SQDG isolated from Porphyridium purpureum and from Spirulina platensis. HSV-1 was tested by a bioassay for anti-herpes simplex virus $[17,62,64]$. Glycolipids extracts isolated from distinct cyanobacteria containing sulfolipids and galactolipids (Table 1) had the capacity to inhibit the polymerase activity of HIV-1 RT [20,39]. The mechanism of the action of GLs remains obscure, but as was stated above, the bioactivity of GLs results from the sugar moiety, the position of the glycerol linkage to the sugar, the length and location of the acyl chain and the anomeric configuration of the sugar [65]. The antiviral activity of SQDGs may be supplied by the sulfonate group [17]. It was postulated that lipophilic groups of SQDG interact with the positive charged side of DNA polymerase. The sulfonate part of SQDG is also important in inhibiting the polymerase activity.

As well as the varied and interesting biological properties of glycolipids, they are also considered a rich source of $n$ - 3 PUFAs essential for human and animal nutrition, being used as food or as additives or supplements $[9,23,54,66]$. The $n-3$ PUFA, particularly EPA and DHA, have beneficial effects at different levels, from fetal development to cancer prevention [67]. The $n-3$ PUFAs have a key role in the prevention of cardiovascular disease, by stabilizing the atherosclerotic plaque and reducing the infiltration of inflammatory and immune cells (lymphocytes and macrophages) into the plaque $[68,69]$. Many chronic conditions, cardiovascular diseases, diabetes, cancer, obesity and autoimmune diseases increase with higher $n-6$ fatty-acid intake but decrease with $n-3$ fatty-acid intake [67,70]. Thus, it is recommended the optimal ratio of $(n-6) /(n-3)$ ranges from $1 / 1$ to $4 / 1$, crucial to reduce the risk of many chronic diseases such as cardiovascular and neurodegenerative diseases [70]. Microalgae are rich in n-3 PUFAs like 18:3, 20:5 or 22:6 FA [69]. In actual fact, successful commercial examples of 
enriched food with PUFAs from marine algae include infant milk formulas and the production of "OMEGA" eggs [54,66,68]. The n-3 FA from polar-lipid rich oil of the microalgae Nannochloropsis oculata with krill oil in rat experiments revealed higher contents of EPA in retroperitoneal adipose tissue, suggesting that MGDGs and DGDGs from microalgae may promote the effective delivery of EPA to plasma and tissues [71]. Few of the microalgae species naturally biosynthesize docosahexaenoic acids (DHA) (e.g., I. galbana), but several strains are able to biosynthesize EPA 20:5, whicht is currently commercialized as a food supplement [23,68,72]. Most of GLs isolated from microalgae contain EPA, particularly Chlorophyta, Bacillariophyta and Eustigmatophyta, an essential nutrient for the production of zooplankton. EPA is used in aquaculture due to its anti-inflammatory properties and in human health due to its recognized benefits [24,26,30,56].

GLs have positive effects on the survival, growth, development, productivity and fertility of animals, boosting their health $[23,66]$. The beneficial properties of GLs, namely as anti-inflammatory promoters $[17,61]$, enhance the use of microalgal biomass enriched in GLs as feed additives, namely in poultry production and aquaculture. The cosmetic and pharmaceutical industries are prominent users of GLs $[8,23,66]$ and it is expected that the next trends in skin care may have formulas enriched with GLs, bringing direct potential benefits to the epidermis, or may use GLs as a carrier of other substances due to their emulsifying properties $[8,23]$.

However, further studies are needed to extend the knowledge concerning the mechanism of action of these molecules as well as their distribution between microalgae species. Glycolipids from microalgae are thus compounds with high biotechnological potential for food and health applications. Due to this fact, the need arises for new research concerning the identification of each particular glycolipid signature of each distinct microalgae lineage, as well as the deviations induced by environmental and growth conditions fostering better understanding of the relation between the microalgae species/structure and associated bioactivity.

Table 1. Glycolipids from microalgae and their potential biological activities.

\begin{tabular}{|c|c|c|c|}
\hline Species Name & Glycolipid & Mechanism & Reference \\
\hline \multicolumn{4}{|c|}{ Antiviral } \\
\hline $\begin{array}{l}\text { Scytonema sp. } \\
\text { Oscillatoria raoi } \\
\text { Oscillatoria trichoides } \\
\text { Oscillatoria limnetica } \\
\text { Phormidium tenue }\end{array}$ & $\begin{array}{l}\text { MGDG } \\
\text { DGDG } \\
\text { SQDG }\end{array}$ & \multirow[t]{2}{*}{ Inhibit HIV-1 RT activity } & [20] \\
\hline Spirulina platensis & SQDG & & [62] \\
\hline \multicolumn{4}{|c|}{ Antitumor } \\
\hline Chlorella vulgaris & $\begin{array}{l}\text { MGDG } \\
\text { DGDG }\end{array}$ & $\begin{array}{l}\text { Antitumor properties expressed by the } \\
\text { inhibitory effects of galactolipids on in vitro } \\
\text { assay of TPA-induced EBV-EA activation } \\
\text { on Raji cells }\end{array}$ & [21] \\
\hline \multicolumn{4}{|c|}{ Anti-proliferative } \\
\hline Porphyridium cruentum & SQDG & $\begin{array}{l}\text { Inhibition of mammalian r-DNA } \\
\text { polymerase } \\
\text { Inhibition of the growth cancer cell-lines on } \\
\text { human colon (DLD-1), breast (MCF-7), } \\
\text { prostate adenocarcinoma (PC-3) and } \\
\text { malignant melanoma (M4 Beu) cancer cells }\end{array}$ & [57] \\
\hline \multicolumn{4}{|c|}{ Antioxidant } \\
\hline Porphyridium cruentum & SQDG & $\begin{array}{l}\text { Inhibition of superoxide generation by } \\
\text { activated peritoneal mono nuclear cells } \\
\text { (PMNs) }\end{array}$ & [57] \\
\hline
\end{tabular}


Table 1. Cont.

\begin{tabular}{|c|c|c|c|}
\hline Species Name & Glycolipid & Mechanism & Reference \\
\hline \multicolumn{4}{|c|}{ Anti-inflammatory } \\
\hline Porphyridium purpureum & SQDG & $\begin{array}{l}\text { Glycolipids inhibited the NO production } \\
\text { through the downregulation of iNOS } \\
\text { expression }\end{array}$ & [64] \\
\hline $\begin{array}{l}\text { Tetraselmis chui } \\
\text { Nannocloropsis granulata } \\
\text { Porphyridium aerugineum }\end{array}$ & $\begin{array}{l}\text { MGDG } \\
\text { DGDG }\end{array}$ & $\begin{array}{l}\text { Glycolipids inhibited the NO production } \\
\text { through the downregulation of iNOS } \\
\text { expression }\end{array}$ & {$[58,59,73]$} \\
\hline Pavlova lutheri & $\begin{array}{l}\text { SQDG } \\
\text { MGDG } \\
\text { DGDG }\end{array}$ & $\begin{array}{l}\text { Downregulation of the production of } \\
\text { cytokine IL-6, IL-8 }\end{array}$ & [74] \\
\hline$M G D G, D G D G, S Q D G$ & $\begin{array}{l}18: 4 / 16: 0 \\
18: 3 / 16: 0\end{array}$ & $\begin{array}{l}\text { Anti-inflammatory effect on croton } \\
\text { oil-induced ear oedema and } \\
\text { carrageenan-induced paw oedema }\end{array}$ & {$[61]$} \\
\hline \multicolumn{4}{|c|}{ Supply n-3 PUFA } \\
\hline Nannocloropsis sp. & $\begin{array}{l}\text { MGDG } \\
\text { DGDG }\end{array}$ & $\begin{array}{l}\text { Glycolipids in the algal oil may promote } \\
\text { effective delivery of EPA to plasma and } \\
\text { tissue }\end{array}$ & [71] \\
\hline
\end{tabular}

Despite of the interesting bioactive properties of GLs (glycolipids), their structure and diversity are far from being entirely recognized, preventing the full exploitation of the biotechnological potential of microalgae.

\section{Lipidomic Approaches of Microalgae}

Lipidomics embraces the detailed and comprehensive identification and quantification of the main molecular species of lipids [75,76]. The lipid analysis is nowadays supported by MS-based approaches that allow the profiling of the total lipid extract obtained from the algae material without chemical modification, requiring relatively small amounts of samples [75]. The glycolipidomic profiling of microalgae requires qualitative and quantitative information on the numerous individual lipid molecular species within each MGDG, DGDG and SQDG classes [32,33,52]. Scarce studies dealing with the glycolipid composition of microalgae have been performed in the past, as indicated in Figure 3 by the number of publications in the study of glycolipids in microalgae in the period 2000-2015.

The majority of the investigations on glycolipids from microalgae used very traditional methods of lipid analysis based on previous separation of polar lipid classes by TLC (thin-layer chromatography) and by silica gel on column chromatography $[19,26,32,52,60,77-80]$. The analysis of the fractionated classes was further performed by GC-MS analysis of the methylated fatty acids $[20,21,49,69,77-79]$. These studies based on previous separation of classes and analysis of the fatty acids profiles were the launch topics on the lipidome of marine microalgae, but lack important information about the specific molecular species. Meanwhile, different techniques based on high-resolution 1D and 2D nuclear magnetic resonance (NMR) spectroscopy $[39,58,59,80-82]$ were applied for structural characterization of isolated GLs from microalgae lipid extracts. The arrival of new modern technologies on mass spectrometry led to the emergence of several works on the lipidome of microalgae, so much that over the last years almost thirty scientific reports have been published (Table 2) $[14,19,24,27,28,52]$. 


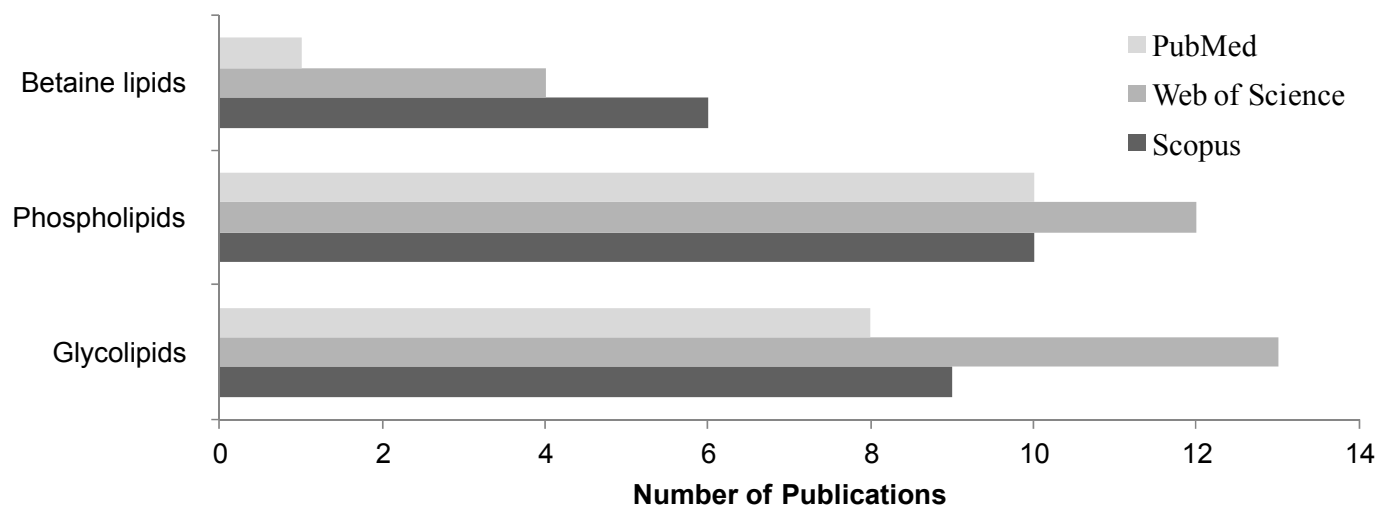

Figure 3. Number of original articles published between 2000 and 2015 searching the terms "glycolipids in microalgae by mass spectrometry/MS"; "phospholipids in microalgae by mass spectrometry/MS" and "betaines in microalgae by mass spectrometry/MS". Citation analysis from Scopus (www.scopus.com) is represented by dark grey columns, Web of Science (http:/ / apps.webofknowledge.com) database correspond to grey columns and light grey columns correspond to the search in PubMed (www.ncbi.nlm.nih.gov/pubmed) accessed on end March of 2016.

MS-based lipidomics platforms are a powerful potential tool in marine polar lipid research due to their relatively simple analytical process and potential for high-throughput analysis [82-85]. These advanced analytical approaches include several steps, starting with obtaining total lipid extracts using organic solvents, or nowadays green solvent approaches, followed by direct analysis by MS or by LC-MS (Figure 4). In between can be included a previous fractionation of the total lipid extract by column chromatography or TLC plates, providing the identification of GLs present in microalgae. The MS-based platform uncovers the overall profile of lipids in a specific physiological state and profile variations depending on the external conditions or alteration of homeostasis.



Figure 4. LC-MS approach in the lipidomic analysis of polar lipids from microalgae. 


\subsection{Lipid Extraction and Isolation of Glycolipids}

In general, lipidomics applications require sample preparation methodology that is fast, reproducible and able to extract a wide range of analytes with different polarities compatible with the instrumental technique. Since many lipid classes consist of hydrophobic moieties (fatty acids) and polar functional groups (galactose, sulfoquinovose), most of the methods referred to in the literature are based on traditional extraction of lipids using conventional methodologies such as Bligh and Dyer $[72,86]$ and Folch $[15,87]$. New methodologies for the extraction and specific isolation of polar lipids from microalgae are being studied [88-91]. Less-toxic solvents substitutes to chloroform such as ethanol, 2-ethoxyethanol, isopropanol and hexane have been investigated. The solvent to be used depends on the class of lipids to be extracted. In a recent research report, the use of 2-ethoxyethanol (2-EE) was shown to provide superior lipid recovery compared to other common extraction solvents, such as chloroform/methanol $[88,92]$. New green methodologies such as supercritical carbon dioxide extraction, pressurized liquid extraction or sonication [90-93] are novel methodologies to extract polar lipids from microalgae that are capable of replacing the traditional chloroform/methanol systems [86,87].

The total lipid extract can then be analyzed by LC-MS allowing identification of the glycolipids. However, the presence of other polar lipids may suppress some of the signals of glycolipids molecular species in the mass spectra. Therefore, several studies use a previous fractionation step of the total lipid extracts in order to obtain isolated fractions. The separation of the glycolipids fraction is usually carried out on chromatographic columns of silica gel, solid-phase extraction (SPE) or by thin layer chromatography (Table 2). TLC is also used for glycolipid analysis, allowing the separation of lipid classes from the total lipid extracts. Each lipid class can be further quantified by colorimetric methods followed by densitometry [26] or further characterized by GC-MS analysis of methyl ester fatty acids (FAMEs) $[21,57,89]$. More recently, extraction of lipids from the spots and further analysis by MS has been performed, giving the detailed structural information on the lipid molecular species within each class [32,33,52]. Quantification of GLs may be carried out by estimation of the sugar content using colorimetric methods such as anthrone and phenol sulfuric acid procedure modified for use with lipids $[63,94]$. These colorimetric methods can be used to estimate the total lipid content in GLs from the total lipid extracts, or to quantify the GL classes fractionated in spots of TLC plates.

Table 2. MS-based approach on the glycolipids composition of microalgae, as reported in literature.

\begin{tabular}{|c|c|c|c|c|c|}
\hline Species Name & MS Approach & Extraction & $\begin{array}{l}\text { Abundant Molecular Species } \\
\text { GL-R } \mathbf{R}_{1} / \mathbf{R}_{2} \text { (Total Number of } \\
\text { Molecular Species) }\end{array}$ & GL\% & Reference \\
\hline \multicolumn{6}{|c|}{ Cyanobacterium } \\
\hline $\begin{array}{l}\text { Spirulina } \\
\text { platensis }\end{array}$ & $\begin{array}{l}\text { LC-MS }^{n} \\
\text { Q-TOF }\end{array}$ & $\begin{array}{c}\text { Ethanol } \\
\text { Pressurized liquid } \\
\text { extraction (PLE) }\end{array}$ & $\begin{array}{l}\text { MGMG 16:0, 16:2, 18:2, 18:3 } \\
\text { SQDG 18:2/16:0, 18:3/16:0 } \\
\text { (4 Lyso-MGDGs, 2 SQDGs) }\end{array}$ & & [93] \\
\hline $\begin{array}{l}\text { Synechocystis } \\
\text { sp. }\end{array}$ & $\begin{array}{l}\text { Off-line TLC } \\
\text { FAB-MS }^{n}\end{array}$ & Bligh and Dyer & $\begin{array}{l}\text { MGDG 18:1/16:0, 18:2/16:0, } \\
\text { 18:3/16:0 } \\
\text { MGMG 16:0 } \\
\text { DGDG 18:1/16:0, 18:2/16:0 } \\
\text { DGMG 16:0 } \\
\text { SQDG 16:1/16:0, 18:1/16:0 } \\
\text { SQMG 16:0 } \\
\text { (4 MGDGs, } 1 \text { MGMG, 3 DGDGs, } 1 \\
\text { DGMG, } 5 \text { SQDGs, } 1 \text { SQMG) }\end{array}$ & & {$[42]$} \\
\hline Scytonema sp. & $\begin{array}{l}\text { Off-line Silica } \\
\text { column } \\
\text { FAB-MS }\end{array}$ & $\begin{array}{l}\text { Methanol/water } \\
\text { Chloroform/methanol }\end{array}$ & $\begin{array}{l}\text { SQTG 16:0/16:0/16:0, } \\
\text { 16:1/16:0/16:0, 18:2/16:0/16:0, } \\
\text { 18:3/16:0/16:0 } \\
\text { (5 SQTGs) }\end{array}$ & & [20] \\
\hline
\end{tabular}


Table 2. Cont.

\begin{tabular}{|c|c|c|c|c|c|}
\hline $\begin{array}{l}\text { Species } \\
\text { Name }\end{array}$ & MS Approach & Extraction & $\begin{array}{c}\text { Abundant Molecular Species } \\
\text { GL-R } \mathbf{R}_{1} / \mathbf{R}_{2} \text { (Total Number of } \\
\text { Molecular Species) }\end{array}$ & GL\% & Reference \\
\hline $\begin{array}{l}\text { Oscillatoria } \\
\quad \text { raoi } \\
\text { Oscillatoria } \\
\text { trichoides }\end{array}$ & $\begin{array}{l}\text { Off-line Silica } \\
\text { column } \\
\text { FAB-MS }\end{array}$ & $\begin{array}{l}\text { Methanol/water } \\
\text { Chloroform/methanol }\end{array}$ & $\begin{array}{l}\text { MGDG 18:1/16:0, 18:2/16:0, } \\
\text { 16:2/16:0 } \\
\text { DGTG 16:1/16:0/16:0, } \\
\text { 18:2/16:0/16:0, 18:3/16:0/16:0 } \\
\text { DGDG 18:1/16:0, 18:2/16:0 } \\
\text { SQDG 16:1/16:0, 16:2/16:0, } \\
\text { 18:1/16:0 } \\
\text { (4 MGTGs,1 DGDG, } 7 \text { MGDGs, } 3 \\
\text { SQDGs) }\end{array}$ & & [20] \\
\hline \multicolumn{6}{|c|}{ Chlorophyta } \\
\hline \multirow[t]{3}{*}{ Chlorella sp. } & $\begin{array}{l}\mathrm{C}_{8} \text { LC-MS }^{n} \\
\text { ESI-Q-TOF }\end{array}$ & Bligh and Dyer & $\begin{array}{l}\text { MGDG 18:3/16:3, 18:4/18:4 } \\
\text { DGDG 20:5/14:0, 20:5/16:0 } \\
\text { SQDG 16:1/16:0 }\end{array}$ & $\begin{array}{l}\text { MDGD } 32 \% \\
\text { DGDG } 6.4 \% \\
\text { SQDG } 36 \%\end{array}$ & [72] \\
\hline & $\begin{array}{l}\text { RP amide } \\
\text { column } \\
\text { LC-MS }^{n} \\
\text { ESI-QqQ }\end{array}$ & Bligh and Dyer & $\begin{array}{l}\text { MGDG 18:2/16:3, 18:3/16:3 } \\
\text { DGDG 18:2/16:3, 18:3/16:3 } \\
\text { SQDG 16:1/16:0 } \\
\text { (14 MGDGs, 16 DGDGs, } 20 \text { SQDG) }\end{array}$ & $\begin{array}{l}\text { MGDG } 42 \% \\
\text { DGDG } 21 \% \\
\text { SQDG 7\% }\end{array}$ & [95] \\
\hline & LC-MS $^{n}$ & $\begin{array}{l}\text { 2-Ethoxyethanol } \\
\text { Hexane and Folch }\end{array}$ & DGDG 18:2/18:2 & & [42] \\
\hline $\begin{array}{l}\text { Chlorella } \\
\text { vulgaris }\end{array}$ & $\begin{array}{c}\text { Off-line SPE-Si } \\
\text { Off-line TLC } \\
\text { LC-MS }^{n} \\
\text { ESI-QqQ }\end{array}$ & $\begin{array}{c}\text { Isopropanol } \\
\text { Chloroform/methanol }\end{array}$ & $\begin{array}{l}\text { MGDG } \Delta \text {-C18/ } \Delta \text {-C16 } \\
\text { DGDG } \Delta-C 18 / \Delta \text {-C16 } \\
\text { SQDG 16:0/16:0 } \\
\text { (13 MGDGs; } 12 \text { DGDGs, 3 SQDGs) }\end{array}$ & & [52] \\
\hline Kyo-Chlorella & $\begin{array}{c}\text { Paper spray } \\
\text { ionization-MS } \\
\text { LTQ-Orbitrap }\end{array}$ & No extraction & $\begin{array}{l}\text { MGDG 16:2/16:3, 18:3/16:3, } \\
\text { 18:3/18:3 } \\
\text { SQDG 18:0/16:0, 18:3/16:0, } \\
\text { 18:3/18:3 } \\
\text { (3 MGDGs, 3 SQDGs) }\end{array}$ & & [96] \\
\hline \multirow[t]{3}{*}{$\begin{array}{l}\text { Chlamydomonas } \\
\text { reinhardtii }\end{array}$} & $\begin{array}{l}\text { Off-line SPE-Si } \\
\text { TLC } \\
\text { LC-MS }^{n} \\
\text { ESI-QqQ }\end{array}$ & $\begin{array}{c}\text { Isopropanol } \\
\text { Chloroform/methanol }\end{array}$ & $\begin{array}{l}\text { MGDG 20:5/14:0 } \\
\text { DGDG 18:1/16:0 } \\
\text { SQDG 16:0/16:0 } \\
\text { (14 MGDGs; } 16 \text { DGDGs, } 6 \text { SQDGs) }\end{array}$ & & [52] \\
\hline & $\begin{array}{l}\text { Nano ESI-MS }{ }^{n} \\
\text { LTQ }\end{array}$ & $\begin{array}{l}\text { Chloroform/methanol/ } \\
\text { water }\end{array}$ & $\begin{array}{l}\text { MGDG 34:7, 36:3, 36:5 } \\
\text { DGDG 34:7, 34:6, 34:1, 34:3, 34:2 } \\
\text { SQDG 32:0, 34:3, 34.2, 34:1 } \\
\text { (4 MGDGs; } 5 \text { DGDGs, } 4 \text { SQDGs) }\end{array}$ & & [34] \\
\hline & $\begin{array}{l}\text { Off line TLC } \\
\text { MALDI-TOF }\end{array}$ & Folch et al. & $\begin{array}{l}\text { MGDG } 36: 8 \text { and } 34: 7 \\
\text { DGDG } 34: 6 \text { and several 34:n, } n=1-7 \\
\text { SQDG } 32: 1 \\
\text { (5 MGDGs, } 9 \text { DGDGs and } 2 \text { SQDGs) }\end{array}$ & & [26] \\
\hline $\begin{array}{c}\text { Clamydomonas } \\
\text { nivalis }\end{array}$ & $\begin{array}{l}\mathrm{C}_{18} \mathrm{LC}^{-\mathrm{MS}^{n}} \\
\mathrm{Q}-\mathrm{TOF}\end{array}$ & Bligh and Dyer & $\begin{array}{l}\text { MGDG 18:3/16:4, 18:3/16:3 } \\
\text { DGDG 18:3/16:4, 18:1/16:3, } \\
\text { 18:2/16:0 } \\
\text { SQDG 16:0/16:0, 16:0/18:3 } \\
\text { (2 MGDGs, 5 DGDGs, 2 SQDGs) } \\
\text { MGDG 18:3/16:2, 18:3/16:3, } \\
\text { 18:1/16:2 } \\
\text { DGDG 18:3/16:0, 18:1/16:3, } \\
\text { 18:2/16:0, 18:1/16:0 } \\
\text { SQDG 16:0/16:0, 16:0/18:3, } \\
\text { 16:0/18:2, 16:0/18:1 } \\
\text { (3MGDGs, } 4 \text { DGDGs, } 4 \text { SQDGs) }\end{array}$ & $\begin{array}{l}\text { MGDG } 41 \% \\
\text { DGDG } 13 \% \\
\text { SQDG } 11 \%\end{array}$ & {$[32]$} \\
\hline $\begin{array}{l}\text { Dunaliella } \\
\text { tertiolecta }\end{array}$ & $\begin{array}{l}\text { ESI-MS }^{n} \\
\text { LTQ }\end{array}$ & Folch et al. & $\begin{array}{l}\text { MGDG 18:3/16:3 } \\
\text { DGDG 18:3/16:4, 18:3/16:3, } \\
\text { 18:3/16:2, 18:4/16:0 } \\
\text { SQDG 16:0/16:0, 18:3/16:0 } \\
\text { 2 MGDGs, 6 DGDGs, } 4 \text { SQDGs) }\end{array}$ & & [15] \\
\hline $\begin{array}{l}\text { Scenedesmus } \\
\text { sp. }\end{array}$ & $\begin{array}{c}\text { Off-line SPE-Si } \\
\text { Off-line TLC } \\
\text { LC-MS }^{n} \\
\text { ESI-QqQ }\end{array}$ & $\begin{array}{c}\text { Isopropanol } \\
\text { Chloroform/methanol }\end{array}$ & $\begin{array}{l}\text { MGDG } \Delta \text {-C18/ } \Delta \text {-C16 } \\
\text { DGDG } \Delta \text {-C18/ }- \text {-C16 } \\
\text { SQDG 16:0/16:0 } \\
\text { (14 MGDGs; } 16 \text { DGDGs, 6 SQDGs) }\end{array}$ & & [52] \\
\hline
\end{tabular}


Table 2. Cont.

\begin{tabular}{|c|c|c|c|c|c|}
\hline Species Name & MS Approach & Extraction & $\begin{array}{c}\text { Abundant Molecular Species } \\
\text { GL-R } \mathbf{R}_{1} / \mathbf{R}_{2} \text { (Total Number of } \\
\text { Molecular Species) }\end{array}$ & GL\% & Reference \\
\hline Tetraselmis chuii & $\begin{array}{l}\mathrm{C}_{18} \text { LC-MS } \\
\text { ESI-Q-TOF }\end{array}$ & Bligh and Dyer & $\begin{array}{l}\text { MGDG 18:1/16:1, 18:1/16:0, } \\
\text { 18:4/16:4, 18:3/16:4 } \\
\text { DGDG 18:1/16:0, 18:2/16:0, } \\
\text { 18:3/16:0 } \\
\text { SQDG 18:1/16:0, 18:3/16:0 } \\
\text { (11 MGDGs, } 7 \text { DGDGs, 16 SQDGs) }\end{array}$ & & [28] \\
\hline \multicolumn{6}{|c|}{ Bacillariophyta } \\
\hline $\begin{array}{l}\text { Chaetoceros } \\
\text { calcitrans }\end{array}$ & $\begin{array}{l}\mathrm{C}_{8} \text { LC-MS }^{n} \\
\text { ESI-Q-TOF }\end{array}$ & Bligh and Dyer & $\begin{array}{l}\text { MGDG 18:3/16:3, 18:4/18:4 } \\
\text { DGDG 20:5/14:0, 20:5/16:0 } \\
\text { SQDG 16:1/16:0 }\end{array}$ & $\begin{array}{l}\text { MGDG } 8 \% \\
\text { DGDG } 4 \% \\
\text { SQDG } 26 \%\end{array}$ & $\begin{array}{l}{[72]} \\
{[97]}\end{array}$ \\
\hline $\begin{array}{c}\text { Cyclotella } \\
\text { meneghiniana }\end{array}$ & $\begin{array}{l}\text { Off line TLC } \\
\text { MALDI-TOF }\end{array}$ & Folch et al. & $\begin{array}{l}\text { MGDG } 36: 6 \text { and } 32: 6 \\
\text { DGDG } 36: 7 \text { and } 32: 1 \\
\text { SQDG 32:1 } \\
\text { (5 MGDGs, } 9 \text { DGDGs, } 2 \text { SQDGs) }\end{array}$ & & [26] \\
\hline Haslea ostrearia & $\begin{array}{l}\text { Off-line Silica } \\
\text { Column } \\
\text { ESI-LTQ-MS }\end{array}$ & Bligh and Dyer & $\begin{array}{l}\text { MGDG 18:3/16:3, 18:3/16:2, } \\
\text { 18:2/16:3 } \\
\text { DGDG 18:3/18:3, 18:3/16:0 } \\
\text { (19 MGDGs, 5 DGDGs) }\end{array}$ & & [98] \\
\hline $\begin{array}{l}\text { Navicula } \\
\text { perminuta }\end{array}$ & $\begin{array}{l}\text { Off-line Silica } \\
\text { Column } \\
\text { ESI-LTQ-MS }\end{array}$ & Bligh and Dyer & $\begin{array}{l}\text { MGDG 18:2/16:0, 18:4/16:0, } \\
\text { 20:5/16:3 } \\
\text { DGDG 18:3/16:3 } \\
\text { (3 MGDGs, } 1 \text { DGDGs) }\end{array}$ & & [98] \\
\hline \multirow[t]{2}{*}{$\begin{array}{l}\text { Phaeodactylum } \\
\text { tricornutum }\end{array}$} & $\begin{array}{l}\text { C }_{8} \text { LC-MS }^{n} \\
\text { ESI-Q-TOF }\end{array}$ & Bligh and Dyer & $\begin{array}{l}\text { MGDG 18:3/16:3, 18:4/18:4, } \\
\text { 16:1/16:0 } \\
\text { DGDG 20:5/14:0, 20:5/16:0 } \\
\text { SQDG 16:1/16:0 } \\
\text { (21 MGDGs, } 9 \text { DGDGs) }\end{array}$ & $\begin{array}{l}\text { MGDG } 5.1 \% \\
\text { DGDG } 3.7 \% \\
\text { SQDG } 26 \%\end{array}$ & [72] \\
\hline & $\begin{array}{l}\text { Off-line Silica } \\
\text { Column } \\
\text { ESI-LTQ-MS }\end{array}$ & Bligh and Dyer & $\begin{array}{l}\text { MDGD 20:5/16:2, 20:5/16:3 } \\
\text { DGDG 16:1/16:1, 16:1/16:0, } \\
\text { 20:5/16:1 } \\
\text { (20 MGDGs, } 9 \text { DGDGs) }\end{array}$ & & [98] \\
\hline $\begin{array}{l}\text { Nitzschia } \\
\text { closterium }\end{array}$ & $\begin{array}{l}\mathrm{C}_{8} \text { LC-MS }^{n} \\
\text { ESI-Q-TOF }\end{array}$ & Bligh and Dyer & $\begin{array}{l}\text { MGDG 20:5/16:2, 20:5/16:3, } \\
\text { 16:2/16:3 } \\
\text { DGDG 20:5/16:2 } \\
\text { SQDG 16:1/14:0 } \\
\text { Lyso SQDG 16:0, 16:1 } \\
\text { (4 MGDGs, } 1 \text { DGDG, } 3 \text { SQDGs, } 3 \\
\text { Lyso-SQDGs) }\end{array}$ & & $\begin{array}{l}{[99]} \\
{[25]}\end{array}$ \\
\hline \multirow[t]{2}{*}{ Skeletonema sp. } & $\begin{array}{l}\text { C } 18_{18} \text { LC-MS } \\
\text { ESI-Q-TOF }\end{array}$ & Bligh and Dyer & $\begin{array}{l}\text { MGDG 16:3/16:3, 20:5/16:1, } \\
\text { 20:5/16:3 } \\
\text { DGDG 20:5/16:1, 20:5/16:2, } \\
\text { 16:1/16:1 } \\
\text { SQDG 14:0/14:0, 14:0/16:0, } \\
\text { 14:0/16:1, 14:0/16:3 } \\
\text { (19 MGDGs, } 9 \text { DGDGs, 22 SQDGs) }\end{array}$ & $\begin{array}{l}\text { MGDG } \\
45-71 \% \\
\text { DGDG } \\
11-49 \% \\
\text { SQDG } \\
11-40 \%\end{array}$ & [24] \\
\hline & $\begin{array}{l}\text { Off-line Silica } \\
\text { Column } \\
\text { ESI-LTQ-MS }\end{array}$ & Bligh and Dyer & $\begin{array}{l}\text { MGDG 16:1/16:3, 16:2/16:2 } \\
\text { DGDG 20:5/16:1 } \\
\text { (3 MGDs,1 DGDGs) }\end{array}$ & & [98] \\
\hline $\begin{array}{c}\text { Stephanodiscus } \\
\text { sp. }\end{array}$ & $\begin{array}{l}\mathrm{C}_{18} \text { LC-MS } \\
\text { ESI-Q-TOF }\end{array}$ & Bligh and Dyer & $\begin{array}{l}\text { MGDG 16:2/16:0, 16:0/16:1 } \\
\text { DGDG 20:5/16:1, 16:1/16:0 } \\
\text { SQDG 14:0/16:0, 16:0/16:1, } \\
\text { 16:2/16:2, 20:5/18:4 } \\
\text { (16 MGDGs, } 9 \text { DGDGs, 23 SQDGs) }\end{array}$ & $\begin{array}{l}\text { MGDG } 68 \% \\
\text { DGDG } 6.3 \% \\
\text { SQDG } 21 \%\end{array}$ & [27] \\
\hline $\begin{array}{c}\text { Thalassiosira } \\
\text { weissflogii }\end{array}$ & $\begin{array}{l}\text { Off-line Silica } \\
\text { Column } \\
\text { ESI-LTQ-MS }\end{array}$ & Bligh and Dyer & $\begin{array}{l}\text { MDGD 14:0/16:1, 16:0/16:3 } \\
\text { DGDG 20:5/16:2 } \\
\text { (5 MGDGs, } 1 \text { DGDGs) }\end{array}$ & & [98] \\
\hline
\end{tabular}


Table 2. Cont.

\begin{tabular}{|c|c|c|c|c|c|}
\hline $\begin{array}{l}\text { Species } \\
\text { Name }\end{array}$ & MS Approach & Extraction & $\begin{array}{l}\text { Abundant Molecular Species } \\
\text { GL-R } \mathbf{R}_{1} / \mathbf{R}_{2} \text { (Total Number of } \\
\text { Molecular Species) }\end{array}$ & GL\% & Reference \\
\hline \multicolumn{6}{|c|}{ Dinoflagellata } \\
\hline \multirow[t]{2}{*}{$\begin{array}{l}\text { Dinoflagellate } \\
\text { spp. }\end{array}$} & $\begin{array}{l}\text { Off-line Silica } \\
\text { Column } \\
\text { ESI-LTQ-MS }^{n}\end{array}$ & Bligh and Dyer & $\begin{array}{l}\text { MGDG 18:5/18:4, 18:5/18:5 } \\
\text { DGDG 18:4/18:4, 18:5/18:4 } \\
\text { (4 MGDG, } 4 \text { DGDG) } \\
\text { MGDG 20:5/18:4, 20:5/18:5 } \\
\text { DGDG 20:5/18:4, 20:5/18:5 } \\
\text { (4 MGDGs, } 4 \text { DGDGs) }\end{array}$ & & [100] \\
\hline & $\begin{array}{l}\text { Off-line Silica } \\
\text { Column } \\
\text { ESI-LTQ-MS }\end{array}$ & \multirow[t]{2}{*}{ Bligh and Dyer } & $\begin{array}{l}\text { MGDG 18:5/18:4, 18:4/18:4, } \\
\text { 18:5/18:5 } \\
\text { DGDG 18:4/18:4, 18:5/18:4, } \\
\text { 18:5/18:5, 18:1/14:0 } \\
\text { TGDG 18:1/14:0, 18:1/16:0, } \\
\text { 18:1/18:1 } \\
\text { (3 MGDGs, } 4 \text { DGDGs, } 3 \text { TGDGs) }\end{array}$ & & [43] \\
\hline $\begin{array}{l}\text { Glenodinium } \\
\text { sanguineum }\end{array}$ & $\begin{array}{l}\mathrm{C}_{18} \text { LC-MS }^{n} \\
\text { ESI-Q-IT }\end{array}$ & & $\begin{array}{l}\text { MGDG 20:5/ } \Delta-C 18,20: 5 / \Delta-C 16, \\
\Delta-C 18 / \Delta-C 16, \Delta-C 18 / \Delta-C 18 \\
\text { DGDG } \Delta-C 18 / \Delta-C 16, \Delta-C 18 / \Delta-C 18 \\
(10 \text { MGDGs,11 DGDGs) }\end{array}$ & & [97] \\
\hline Pyrocystis sp. & $\begin{array}{l}\text { Off-line Silica } \\
\text { Column } \\
\text { ESI-LTQ-MS }^{n}\end{array}$ & Bligh and Dyer & $\begin{array}{l}\text { MGDG 20:5/18:5, 20:5/18:4 } \\
\text { DGDG 20:5/18:5, 20:5/18:4 } \\
\text { TGDG 18:1/14:0, 22:6/16:0 } \\
\text { (2MGDGs, 2DGDGs, 2 TGDGs) }\end{array}$ & & [101] \\
\hline \multicolumn{6}{|c|}{ Eustigmatophyta } \\
\hline \multirow[t]{2}{*}{$\begin{array}{l}\text { Nannochloropsis } \\
\text { oculata }\end{array}$} & $\begin{array}{l}\mathrm{C}_{8} \text { LC-MS }^{n} \\
\text { ESI-Q-TOF }\end{array}$ & Bligh and Dyer & $\begin{array}{l}\text { MGDG 18:3/16:3, 18:4/18:4 } \\
\text { DGDG 20:5/14:0, 20:5/16:0 } \\
\text { SQDG 16:1/16:0 }\end{array}$ & $\begin{array}{l}\text { MGDG } 17 \% \\
\text { DGDG } 12 \% \\
\text { SQDG } 6.3 \%\end{array}$ & {$[72]$} \\
\hline & $\begin{array}{l}\text { nLC-MS }^{n} \\
\text { ESI FT-ICR }\end{array}$ & Chloroform/methanol & $\begin{array}{l}\text { MGDG 20:5/20:5 } \\
\text { DGDG 20:5/16:0, 20:5/16:1 } \\
\text { SQDG 16:1/16:0 } \\
\text { (13 MGDGs, 20 DGDGs, } 33 \text { SQDGs }\end{array}$ & & {$[14]$} \\
\hline $\begin{array}{l}\text { Nannocloropsis } \\
\text { oceanica }\end{array}$ & $\begin{array}{l}\mathrm{C}_{8} \text { LC-MS } \\
\text { ESI-Q-TOF }\end{array}$ & Bligh and Dyer & $\begin{array}{l}\text { MGDG 20:5/14:0, 20:5/16:0, } \\
\Delta \text {-C16/ } \Delta \text {-C16, } \Delta \text {-C18/ } \Delta \text {-C16 } \\
\text { DGDG 20:5/16:0, } \Delta \text {-C18/ } \Delta \text {-C16 } \\
\text { SQDG } \Delta-C 16 / \Delta-C 16,20: 5 / 16: 0, \\
\text { (6 MGDGs, } 4 \text { DGDGs, } 7 \text { SQDGs) }\end{array}$ & & [102] \\
\hline \multirow[t]{4}{*}{$\begin{array}{l}\text { Nannochloropsis } \\
\text { sp. }\end{array}$} & $\begin{array}{l}\text { Off-line SPE-Si } \\
\text { Off-line TLC } \\
\text { LC-MS }^{n} \\
\text { ESI-QqQ }\end{array}$ & $\begin{array}{c}\text { Isopropanol } \\
\text { Chloroform/methanol }\end{array}$ & $\begin{array}{l}\text { MGDG 20:5/14:0, } \\
\text { DGDG 20:5/14:0, 20:5/16:0, } \\
\text { 20:5/16:0 } \\
\text { SQDG 16:1/16:0 } \\
\text { (14 MGDGs; 16 DGDGs, 6 SQDGs) }\end{array}$ & & [52] \\
\hline & $\begin{array}{l}\mathrm{C}_{8} \text { LC-MS }^{n} \\
\text { ESI-Q-TOF }\end{array}$ & Bligh and Dyer & $\begin{array}{l}\text { MGDG 18:3/16:3, 18:4/18:4 } \\
\text { DGDG 20:5/14:0, 20:5/16:0 } \\
\text { SQDG 16:1/16:0 }\end{array}$ & $\begin{array}{l}\text { MGDG } 13 \% \\
\text { DGDG } 5.6 \% \\
\text { SQDG } 18 \%\end{array}$ & [72] \\
\hline & $\begin{array}{l}\text { LC-MSn }^{\text {LC-MqQ }} \\
\text { ESI-QQ }\end{array}$ & Bligh and Dyer & $\begin{array}{l}\text { MGDG 20:5/14:0 } \\
\text { DGDG 20:5/14:0 } \\
\text { SQDG 18:3/16:0 } \\
\text { (14 MGDGs, 16 DGDGs, 20 SQDGs) }\end{array}$ & $\begin{array}{l}\text { MGDG } 42 \% \\
\text { DGDG } 14 \% \\
\text { SQDG } 39 \%\end{array}$ & [95] \\
\hline & $\begin{array}{c}\text { Paper spray } \\
\text { ionization } \\
\text { LTQ-Orbitrap-MS }^{n}\end{array}$ & $\begin{array}{l}\text { No need of } \\
\text { extraction }\end{array}$ & $\begin{array}{l}\text { MGDG 32:3, 32:5, 34:2, 34:6 } \\
\text { SQDG 30:0, 32:1, 34:3, 36:6 } \\
\text { (4 MGDGs, } 4 \text { SQDGs) }\end{array}$ & & [96] \\
\hline \multicolumn{6}{|c|}{ Labyrinthulomycetes } \\
\hline $\begin{array}{l}\text { Schizochytrium } \\
\text { limacinum }\end{array}$ & $\begin{array}{l}\text { Off-line SPE-Si } \\
\text { Off-line TLC } \\
\text { LC-MS }^{n} \\
\text { ESI-QqQ }\end{array}$ & $\begin{array}{c}\text { Isopropanol } \\
\text { Chloroform/methanol }\end{array}$ & $\begin{array}{l}\text { MGDG 22:6/16:0 } \\
\text { DGDG 18:1/18:0 } \\
\text { SQDG 16:1/16:0 } \\
\text { (14 MGDGs; } 16 \text { DGDGs, } 6 \text { SQDGs) }\end{array}$ & & [52] \\
\hline
\end{tabular}


Table 2. Cont.

\begin{tabular}{|c|c|c|c|c|c|}
\hline Species Name & MS Approach & Extraction & $\begin{array}{c}\text { Abundant Molecular Species } \\
\text { GL-R } 1 / \mathbf{R}_{2} \text { (Total Number of } \\
\text { Molecular Species) }\end{array}$ & GL\% & Reference \\
\hline \multicolumn{6}{|c|}{ Rhodophyta } \\
\hline $\begin{array}{l}\text { Porphyridium } \\
\text { purpuream }\end{array}$ & $\begin{array}{l}\text { Off Line TLC } \\
\text { MALDI-QIT-TOF-MS }\end{array}$ & $\begin{array}{l}\text { Dichloromethane/ } \\
\text { methanol }\end{array}$ & $\begin{array}{l}\text { SQDG 18:2/16:0, 20:4/16:0, } \\
\text { 20:5/16:0 (3 SQDGs) }\end{array}$ & & [19] \\
\hline $\begin{array}{l}\text { Porphyridium } \\
\text { aerugineum }\end{array}$ & $\begin{array}{l}\text { Off-line Silica } \\
\text { Column } \\
\text { LC-Q-MS }^{n}\end{array}$ & $\begin{array}{l}\text { Methanol } \\
\text { Ethyl acetate }\end{array}$ & $\begin{array}{l}\text { MGDG 20:4/18:3, 20:4/16:0, } \\
\text { 20:5/16:0, 20:5/18:3, 20:5/20:4 } \\
\text { (5 MGDGs) }\end{array}$ & & [73] \\
\hline \multicolumn{6}{|c|}{ Prymnesiophyta } \\
\hline $\begin{array}{l}\text { Isochrysis } \\
\text { galbana }\end{array}$ & $\begin{array}{l}\mathrm{C}_{8} \text { LC-MS }^{n} \\
\text { ESI-Q-TOF }\end{array}$ & Bligh and Dyer & $\begin{array}{l}\text { MGDG 18:3/16:3, 18:4/18:4, } \\
\text { 22:6/16:0 } \\
\text { DGDG 20:5/14:0, 20:5/16:0 } \\
\text { SQDG 16:1/16:0 }\end{array}$ & $\begin{array}{l}\text { MGDG } 37 \% \\
\text { DGDG } 11 \% \\
\text { SQDG } 41 \%\end{array}$ & [72] \\
\hline
\end{tabular}

MGDG, monogalactosyl diacylglycerol; DGDG, digalactosyl diacylglycerol; TGDG, trigalactosyl diacylglycerol; DGTG, digalactosyl triacylglycerol, with one of the fatty acids linked to the sugar moiety; MGMG, monogalactosyl monoacylglycerol; SQDG, sulfoquinovosyl diacylglycerol; SQTG, sulfoquinovosyl triacylglycerol, with one of the fatty acids linked to the sugar moiety; SQMG, sulfoquinovosylmonoacylglycerol; $\mathrm{C}: \mathrm{N}$, total number of the carbons of the fatty acids: total number of double bonds; $\Delta$, degree of unsaturation ranging from 1 to 4 double bonds; TLC, Thin Layer Chromatography; SPE, Solid-Phase Extraction; Si, Silica; FAB, Fast Atom Bombardment; LC-ESI/FT-ICR-MS, Liquid Chromatography-Electrospray Ionization Fourier Transform Ion Cyclotron Resonance Mass Spectrometry; UPLC, Ultra Performance Liquid Chromatography; LTQ, Linear Ion Trap; Q-IT, Quadrupole-Ion Trap; QqQ, Triple Quadrupole; Q-TOF, Quadrupole-Time of Flight; MALDI, Matrix-Assisted Laser Desorption Ionization.

\subsection{Mass Spectrometry Analysis}

Among the possible mass spectrometry-based approaches, two main strategies for the analysis of polar lipids have been used in most of the described reports: on one hand there is shotgun lipidomics, which relies on a direct infusion analysis of a total lipid extract in mass spectrometers $[15,75,76]$, or by using LC-MS strategy [24,25]. Both methods have their advantages and disadvantages. While the shotgun approach is prone to strong ion suppression effects, this in part can be compensated for by large sample dilutions or by using internal reference compounds. Chromatography-based methods (on-line HPLC-MS, on-line UPLC-MS) avoid suppression effects, resolve problems such as the differentiation of isobaric species and allow exact lipid identification due to the chromatographic separation [85,103].

Recently, new LC-MS platforms have greatly improved the analysis of glycolipids [29,52]. The separation of lipid classes through high or ultra-performance liquid chromatography (HPLC or UPLC) is now most often used [20,24,32,52]. Usually, reversed-phase (RP $C_{8}$ and $\left.C_{18}\right)$ and hydrophilic interaction liquid chromatography (HILIC) columns are used in lipidomic approaches [85,103-105]. HILIC can separate lipid molecular species from different lipid classes from the complex extract of algae. However, in the majority of published works, RP has been most widely used for the analysis of complex lipids as it can distinguish polar lipids supported by their hydrophobic properties based on the number of carbons and the degree of saturation of fatty acyl substituents (Table 2). In fact, separating lipids according to the two independent molecular properties offers great opportunities towards further unscrambling the lipidome complex.

By LC-MS and MS/MS data interpretation it is possible to identify a large number of molecular species from the polar lipid classes in the total lipid extract [14,52]. The identification of the molecular ions is based on the detailed analysis of the MS spectra, and the confirmation of the identity of each molecular species is done by the analysis of MS/MS fragmentation. Unlike phospholipids, there are scarce databases including glycolipids mass spectral data $[34,105,106]$. LipidBlast is a database available to identify individual lipid molecular species by matching mass precursor ions and MS/MS fragmentations. However, there are several drawbacks when using this bioinformatic tool, such as the low mass accuracy of the instrument, isobaric interferences and ion suppression $[105,106]$. Moreover 
individual analysis of the fragmentation pattern of each species is usually needed in order to identify unequivocally the molecular species of glycolipids [107-110], as will be explained in the next section.

MS and LC-MS-based analytical tools are powerful for qualitative but also for quantitative analysis of the glycolipidome $[15,24,25]$. After the identification of molecular species and lipid classes, semi-quantitation of lipid species is achieved by the normalization of the individual molecular ion-peak intensity using an internal standard of each lipid class. The calculated ratio of analyte and internal standard is multiplied by the concentration of the internal standard to obtain the concentration of a particular analyte [85]. The sum of each peak area related with the standard representing each class gives the relative abundance of the different classes of GLs. However, the major drawback results from the unavailability and lack of stability/prohibitive price of reference standards of all glycolipids molecular species. Reviewing the lipidomics papers cited, those including quantitation used at least one internal standard per lipid class $[24,25]$.

Analysis of Glycolipids by Mass Spectrometry

In the field of microalgae, great improvement to lipidomics has been made by the development of soft ionization methods such as electrospray ionization (ESI) [28]. Mass spectrometers with different analyzers, for instance ion trap (IT), orbitrap, quadrupole (Q) and time-of-flight (TOF), have been used for the structural study of polar lipids $[26,27,34,38]$.

Glycolipids can be analyzed by MS in positive and negative modes attending the nature of their structural features [75,107-110]. Neutral GLs are usually identified in MS in positive mode as protonated molecules $[\mathrm{M}+\mathrm{H}]^{+}$, ammonium adducts $\left[\mathrm{M}+\mathrm{NH}_{4}\right]^{+}$or adducts with alkali cations $\left(\mathrm{Na}^{+}\right.$or $\left.\mathrm{Li}^{+}\right),[\mathrm{M}+\mathrm{Na}]^{+}$or $[\mathrm{M}+\mathrm{Li}]^{+}$, while SQDG are mainly detected as negative ions $[\mathrm{M}-\mathrm{H}]^{-}[15,28,107-112]$. The structural features of each molecular species are obtained by the identification of the fragmentation pattern observed in the MS/MS spectra that is unique for each species [107]. These specific fragmentation pathways allow the elucidation of fatty acyl and heading group composition of GLs [19,32]. The detailed structural features obtained from the MS/MS of GLs will be briefly described. The monogalactosyl diglycerides and digalactosyl diglycerides (MGDG and DGDG) are usually detected and fragmented as the ammonium adduct or alkali metal adducts (Figure 5) [42,48,110].
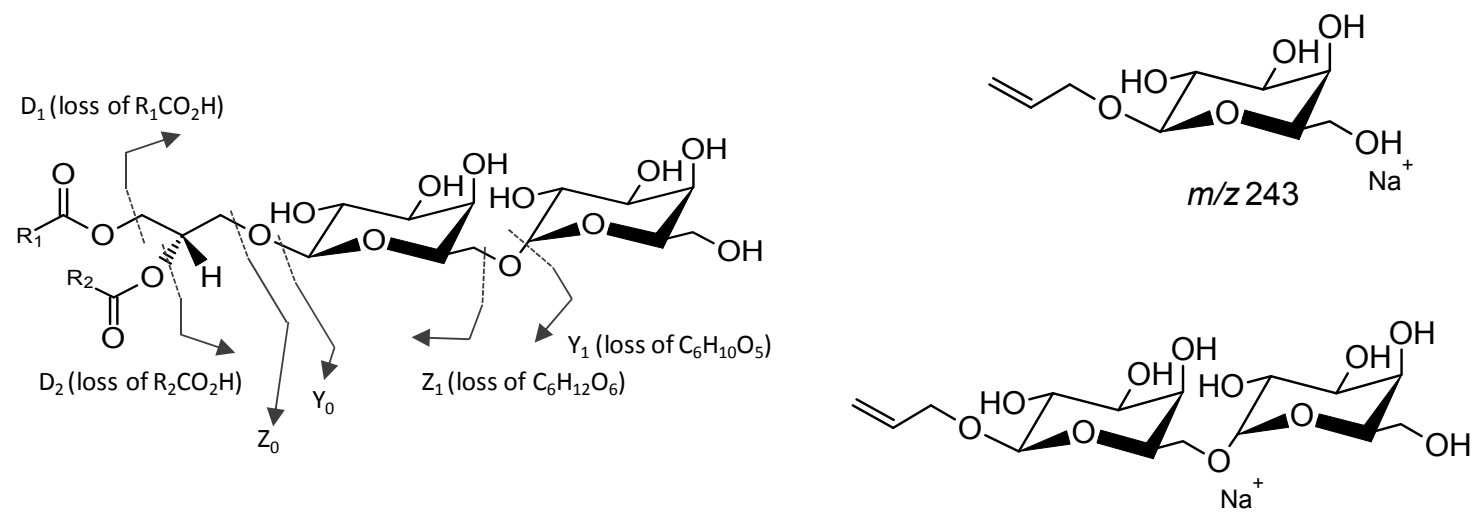

$m / z 405$

Figure 5. Main fragmentation pathways of digalactosyl diacylglycerol (DGDG) observed in the MS/MS spectra of the $[\mathrm{M}+\mathrm{Na}]^{+}$ions that allow the elucidation of structural features. Structures attributed to the product ions at $m / z 243$ and $m / z 405$ are depicted.

The detailed fragmentation of the precursor ion $[\mathrm{M}+\mathrm{Na}]^{+}$yields the product ion galactosyl glycerol head group $\left[\mathrm{C}_{9} \mathrm{H}_{16} \mathrm{O}_{6}+\mathrm{Na}\right]^{+}$at $m / z 243$ (MGDG and DGDG) and the digalactosyl glycerol head group $\left[\mathrm{C}_{15} \mathrm{H}_{26} \mathrm{O}_{11}+\mathrm{Na}\right]^{+}$at $m / z 405$ (DGDG) (Figure 5) [42,48,107-110]. In the case of MGDG, the neutral loss $(\mathrm{NL})$ of a hexose residue $\left(\mathrm{Y}_{1}\right.$, Figure 5$)$ or $\mathrm{NL}$ of a hexose unit $\left(\mathrm{Z}_{1}\right.$, Figure 5$)$ in the 
structure of MGDG allows the confident assignment of the class. Otherwise, the loss of the hexose residues $\left(\mathrm{Y}_{1}, \mathrm{Y}_{0}\right.$, Figure 5$)$ or the loss of hexose units $\left(\mathrm{Z}_{0}\right.$, Figure 5$)$ allow to confirm the DGDG class. The information of fatty acid substituents and their position on the glycerol backbone is obtained by the identification of the product formed due to the loss of the fatty acyl moieties, described by the product ions $\mathrm{D}_{1}$ (loss of the $\mathrm{R}_{1} \mathrm{COOH}$ ) and $\mathrm{D}_{2}$ (loss of the $\mathrm{R}_{2} \mathrm{COOH}$ ). The regiospecificity of the two acyl chains in MGDG and DGDG is usually determined based on the ratio of the relative abundance of the loss of $\mathrm{RCOOH}$ from the $s n-1$ and $s n-2$ product ions attending that the loss of $\mathrm{R}_{1} \mathrm{COOH}$ is more abundant than the loss of $\mathrm{R}_{2} \mathrm{COOH}$ [97]. Also, the fragmentation of GLs observed in the MS/MS spectra as [M + $\left.\mathrm{NH}_{4}\right]^{+}$showed typical product ions due to loss of $\mathrm{NH}_{3}$, loss of hexose residue (-162 Da, -Hex res) and hexose moiety (-180 Da,-Hex) $[109,113]$. Product ions formed by the combined loss of one FA and hexose yield typical acylium ions plus $74(\mathrm{RCO}+74)^{+}$that confirms the FA composition $[110,113]$.

SQDG preferably ionize in negative mode as $[\mathrm{M}-\mathrm{H}]^{-}$due to the strongly acidic character of the sulfonic acid. The MS/MS spectra showed a typical product ion at $m / z 225$ attributed to the sulfoquinovosyl anion $\left[\mathrm{C}_{6} \mathrm{H}_{9} \mathrm{O}_{7} \mathrm{~S}\right]^{-}$, often used as a diagnostic of the presence of SQDG $[24,109,112]$. The neutral loss of each fatty acyl group as a ketene or free carboxylic acid (product ions $\mathrm{D}_{1}$ and $D_{2}$, Figure 6) are typically observed as reasonably abundant ions. However there is not sufficient knowledge of the regioisomeric structure and specific fatty acyl groups to determine $s n-1 / s n-2$ fatty acyl positions [97]. The product ions attributed to carboxylate anions $\left(\mathrm{RCOO}^{-}\right)$can also be seen [97].

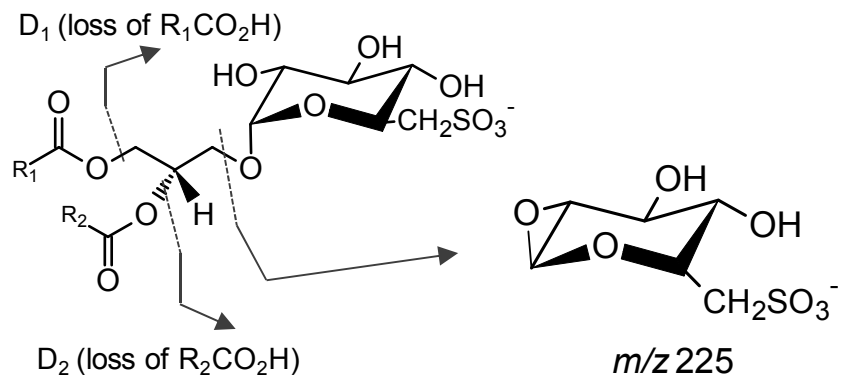

Figure 6. Main fragmentation pathways of SQDG observed in the MS/MS spectra of the $[\mathrm{M}-\mathrm{H}]^{-}$ ion. The lines indicate the product ions formed. The product ion at $m / z 225$ is attributed to the sulfoquinovose head group.

\subsection{Studies Uncovering the Lipidome of Microalgae}

Microalgae mainly comprise photoautotrophic bacteria and distinct microalgae species from phyla Bacillaryophyta (diatoms), Dinophyceae (dinoflagellates), Chlorophyta and Eustigmatophyta, among others. However, only a few studies have reported the lipidomic profile of distinct microalgae. This is the case with Chlamydomonas sp., Chlorella sp., Porphyridium purpuream, Nannochloropsis sp., Dunaliella tertiolecta, Isochrysis galbana, Chaetoceros calcitrans, Cyclotella meneghiniana, Phaeodactylum tricornutum, Stephanodiscus sp., Spirulina platensis, Scenedesmus sp., Tetraselmis chuii, Nitzschia closterium and Schizochytrium limacium, which only recently have been investigated using MS-based approaches (Table 2). The next sections pinpoint the current research in the lipidome of Cyanobacteria, Chlorophyta, Bacyllaryophyta, Dinoflagellata, Eustigmatophyta, Prymnesiophyta and Rodophyta microalgae.

\subsubsection{Glycolipidomic Profiling on Cyanobacteria}

Cyanobacteria are photosynthetic prokaryotes occurring in the phytoplankton. The lipidome of Synechocystis sp., Scytonema sp., Oscillatoria raoi and Spirulina sp. have been characterized [20,42,93]. The lipidic extract of Synechocystis sp. was studied by fast atom bombardment FAB-MS and MS/MS, enhancing for the first time a direct and rapid structural identification of a few glycerolipids at the molecular level [42]. The total lipids of the harvested microalgae were extracted by the method of Bligh and Dyer and the glycolipids were fractioned by two-dimensional thin-layer chromatography 
(2D-TLC), allowing the separation of six classes of glycolipids (14 molecular species) and two classes of phospholipids. The glycolipids of each of the spots were identified by FAB-MS as $[\mathrm{M}+\mathrm{Na}]^{+}$ions correspondent to MGDG, DGDG and SQDG with 16:0, 18:1, 18:2 and 18:3 FA and MGMG, DGMG and SQMG with 16:0 FA. Regarding Scytonema sp. and Oscillatoria raoi [20], besides the glycolipids MGDG, DGDG and SQDG, novel acylated glycolipids of SQDG and DGDG were assigned for the first time in the lipidome of microalgae by MS (Table 2).

The lipidome of Spirulina sp., the most commercialized microalgae used as food [93], was studied by LC-MS analysis of the ethanolic extract obtained using pressurized liquid extraction (PLE). This study allowed the identification of six GLs, namely MGMG (16:0), MGMG (16:2), MGMG (18:2), MGMG (18:3) and SQDG (18:3/16:0) and SQDG (18:2/16:0). The cyanobacteria were found to contain lyso-forms of glycolipids and preferably produce $C_{16}$ and $C_{18}$ PUFA esterified to polar lipids and do not biosynthesize EPA or DHA.

\subsubsection{Glycolipidomic Profiling on Chlorophyta}

Green microalgae Chlorella, Dunaliella and Clamydomonas spp. are widely commercialized microalgae. Most of the recent published works were focused on the lipidome of Chlorella sp., highly prized for commercial applications for food consumption and for feed $[42,52,72,95,96]$. The polar lipids of the Chlorella were analyzed by LC-MS/MS-based approaches [28,52,95]. The lipidome of Chlorella was fully characterized by Yao et al. (2015), and the MGDG (13 molecular species), DGDG (12 molecular species) and SQDG (three molecular species) were found to represent about $79 \%$ of the total polar lipids [52]. Chlorella's polar lipid fraction contained a high amount of PUFA, including 16:3 $(13 \%), 18: 2(23 \%)$ and 18:3 (21\%). Regarding the lifecycle of Chlorella, polar lipids have the highest yield at the onset of the stationary phase, containing MGDG (32\%) with the dominant components MGDG (18:3/16:3) and MDGD (18:4/18:4). SQDG corresponded to about 36\% of the total extract and contained SQDG (16:1/16:0) abundant species. DGDGs (6\%) mainly contained DGDG (20:5/14:0) and DGDG (20:5/16:0) [72].

GLs profile of Chlamydomonas were also studied by LC-MS (Table 2) [26,32-34,52]. The MGDG (14 molecular species), DGDG (16 molecular species) and SQDG (three molecular species) moieties represented $96 \%$ of the total polar lipids (10\% of the total extract). For Chlamydomonas, DGDG and MGDG contained distinct isoforms with 34 carbons and 1-6 double bonds distributed in two acyl groups, which covers all of the $\mathrm{C}_{16}$ and $\mathrm{C}_{18}$ major fatty acids in Chlamydomonas extract.

More than 40 lipid molecular species distributed by the three subclasses MGDG, DGDG and SQDG were identified for the microalgae Tetraselmis chuii [28]. The detailed analysis of the tandem mass spectra of the sodium adducts of each GL molecular species allowed pinpointing the position of the two fatty acids acyl chains of MGDG and DGDG. For the 11 MGDG molecular species, the fatty acids at the $s n-1$ position were 18:1,18:2, 18:3 and 18:4, and the fatty acids at the $s n$-2 position were 16:0, 16:1, 16:2, 16:3 and 16:4. The major molecular species identified were MGDG (18:1/16:1), MGDG (18:1/16:0), MGDG (18:4/16:4) and MGDG (18:3/16:4). For the seven DGDG molecular species, the fatty acids assigned to the $s n-1$ position were 18:1, 18:2 and 18:3 FA. In the 16 SQDG molecular species, the preferential fatty acid at the $s n-2$ position was assigned as $16: 0$, while the fatty acids at the $s n-1$ position included $C_{14-20}$ fatty acids with different unsaturation and hydroxylation. The major SQDG were attributed to SQDG (18:1/16:0) and SQDG (18:3/16:0), and other SQDG molecules, such as SQDG (20:1/16:0) and SQDG (HO-18:3/16:0), were found in significant quantities [28]. Moreover, the study of the lipidome of Scenedesmus sp. by LC-MS allowed the identification of about 46 molecular species as MGDG (14 molecular species), DGDG (16 molecular species) and SQDG (six molecular species), which represented $67 \%$ of the total polar lipids [52]. The GLs were rich in 16:0 (31\%), 16:4 (6\%), 18:1 $(11 \%), 18: 2(11 \%)$ and $18: 3(21 \%)$ and in the unusual $n-3$ fatty acid $18: 4(4 \%)$. The FA $18: 4$ is as effective as marine oil-derived long-chain $n-3$ fatty acids in providing the health benefits in humans associated with cardiovascular disease. In general, SQDG have preferentially saturated fatty acids (SFA) and monounsaturated fatty acids (MUFA), whereas MGDG and DGDG are mainly linked to PUFA. 
Also, the commercial strain Dunaliella sp. [15] was profiled by means of polar lipids using MS-based approaches. Among several polar lipids, the classes MGDG (two molecular species), DGDG (six molecular species) and SQDG (four molecular species) were identified in the lipidome of Dunaliella tertiolecta. Interestingly, a lower number of molecular species were identified in comparison with the ones reported for the Chlorella or Scenedesmus lipidomes [52]. The analyses of the effect of the incidence of light and nitrogen deficiency was evaluated during the stationary phase of Dunaliella tertiolecta [15] and a significant increase of DGDG molecular species containing unsaturated $C_{16}$ fatty acids were detected after both nitrogen starvation and high light intensity. In high light conditions, DGDG molecular species with saturated fatty acid 16:0 showed a significant decline. Nitrate deficiency did not lead to any significant changes in SQDG molecular species, while cultivation under a high light intensity resulted in an increase of SQDG (18:1/16:0) and SQDG (16:0/16:0). Under both effects, SQDG incremented in D. tertiolecta. SQDG has an important role in maintaining function and structure of the photosynthetic apparatus complex, especially under stress conditions such as high temperature [15]. MGDG molecular species did not exhibit variations under stress conditions.

The evaluation of the effect of nutrient limitation on Chlorella under $N$ deprivation led to decreases in MGDG and DGDG (66\%-78\%) and in SQDG (40\%-67\%) contents [95]. MGDG, DGDG and SQDG contained 14, 16 and 20 molecular species, respectively. Analogous effect was found in the genus Clamydomonas sp. [32-34] that under $N$ deprivation, showed a decrease of MGDG and DGDG species, containing 16:0, 18:1, 16:3, 16:4 and 18:3 (Table 2). The Pi starvation also induced some effect on GLs of Chlamydomonas, causing an increase in the amount of DGDG and SQDG species [10,33]. In response to changes in salinity, there was an increase in the amount of glycolipids in Chlamydomonas, mainly expressed by the increase in DGDGs content [32]. Under salt stress, there was a remarkable increase of SQDG and DGDG/MGDG ratio that is proportional to the concentration of $\mathrm{NaCl}$, followed by the increase of unsaturated molecular species. DGDGs exhibit bilayer-forming properties, while MGDG are always non-bilayer forming, and the ratio DGDG/MGDG is required for the maintenance of membrane stability and functionality [32]. The GL signature of microalgae under distinct stress conditions was accomplished by using MS-approaches.

Among the distinct studies profiling the lipidome of microalgae, the reviewed studies support the fact that green algae preferably produce a typical signature and contain high unsaturated $C_{16}$ and $\mathrm{C}_{18}$ FA esters bonded to glycolipids. About fifty molecular species of glycolipids were identified in the lipidome of green algae.

\subsubsection{Glycolipidomic Profiling on Bacillariophyta}

Bacillariophyta or diatoms are single-cell eukaryotic microalgae with promising application in nanotechnology and biotechnology, including nanofabrication techniques, chemo and biosensing and control of particles in nanofluidics systems [114]. These microalgae have also potential to be used in analyzing ecological problems such as climate change, acidification and eutrophication of aqueous ecosystems [114]. Lipid profiling of distinct marine diatoms has been reported for the diatoms Skeletonemas sp., Thalassiosira weissflogii, Stephanodiscus sp., P. tricornutum, Haslea ostrearia, Navicula perminuta and Nitzschia closterium.

Likewise, methanol and chloroform/methanol/water fractions obtained from three strains of Skeletonemas sp. were analyzed by LC-MS to profile the glycolipids [24]. Overall, 19 MGDGs ( $45 \%-70 \%$ of lipidome), nine DGDGs ( $5 \%-15 \%$ of lipidome) and 22 SQDGs $(10 \%-40 \%)$ have been unequivocally identified in the three strains of Skeletonemas sp. The predominant molecular species of MGDG and DGDG contained 16:1, 16:2, 16:3 and 20:5 FA (Table 2) and the major molecular species of SQDG combine 14:0, 16:0, 16:1 and 16:3 FA [24]. Similar molecular species were found in Stephanodiscus sp. lipidome, which showed 16 MGDGs, nine 9 DGDGs and 23 SQDGs [27]. Dodson et al. (2013) compared the GL profiles of two centric diatoms, Skeletonema marinoi and Thalassiosira weissflogii, and the pennate diatoms, P. tricornutum, H. ostrearia and Navicula perminuta, characterized by ESI-MS ${ }^{n}$ approaches [98]. Skeletonema marinoi, Thalassiosira weissflogii and P. tricornutum contained MGDG and 
DGDG predominantly with $\mathrm{C}_{16}, \mathrm{C}_{18}$ and $\mathrm{C}_{20} \mathrm{FA}$. However, this was not observed within all strains and the pennate diatoms $H$. ostrearia and Navicula perminuta contained primarily $C_{18} / C_{16}$ or $C_{18} / C_{18}$ forms of MGDG and DGDG, indicating an unrecognized fatty acid diversity in GLs from diatoms that needs more research to be understood [98].

LC-MS was used to study the changes of the lipidome between genera and species, and also to evaluate the effect of distinct growing stages of microalgae. At the stationary phase, the glycolipidome of P. tricornutum and C. calcitrans enclosed abundant MGDG (18:3/16:3), MGDG (18:4/18:4), DGDG (20:5/14:0), DGDG (20:5/16:0) and SQDG (16:1/16:0) molecular species [72]. The different content of GLs allowed the differentiation of strains [72]. During the growth phase of Nitzschia closterium, glycolipids were found to decrease from the exponential to the stationary phase. However, at the end of the stationary phase, the levels increased due to the role of GLs in the protection of microalgae during the growth phase [99]. The molecular species MGDG (20:5/16:2), MGDG (20:5/16:3) and MGDG (16:2/16:3), DGDG (20:5/16:1), SQDGs (16:1/14:0) and lyso-SQDGs 16:0 and 16:1 are potential biomarkers of the growth cycle [99]. The examples mentioned above enhance the usefulness of MS/MS in profiling GL-rich lipids for artificial rearing of marine organisms. Based on the positional distribution of fatty acids on the individual lipid class of the diatoms, it was indicated that MGDG and DGDG were biosynthesized through the "prokaryotic" pathway exclusively within the chloroplast, because all the fatty acids linked at the $s n-2$ position in MGDG and DGDG were $\mathrm{C}_{16}$ FAs. Additionally, SQDG had a typical mixed biosynthetic pathway (both "prokaryotic" and "eukaryotic") because the fatty acid at the $s n-2$ position was either $\mathrm{C}_{16}$ or $\mathrm{C}_{18}$ fatty acids. However, some pinnate diatoms were found to be able to biosynthesize $C_{18} / C_{16}$ or $C_{18} / C_{18}$ forms of MGDG and DGDG lipids [98]. Several species of diatoms were characterized by MS approaches and more than fifty molecular species are known in the signature of glycolipids. However, the distinct ability to biosynthesize $\mathrm{C}_{18}$ and $\mathrm{C}_{20}$ species is far from being elucidated. Certainly, some species of diatoms are prone to biosynthesize EPA (20:5) $[24,72,98,99]$.

\subsubsection{Glycolipidomic Profiling on Dinoflagellata}

Despite their importance in marine and freshwater microalgal assemblages, dinoflagellates have been the subject of few comprehensive lipid studies, particularly with respect to those lipids that comprise plastid membranes. An effort is being made to understand the differences between dinoflagellate composition in glycolipids by MS [97]. Glenodinium sanguineum was analyzed by LC-MS to demonstrate the correct fatty acid composition and also provide a reliable determination of the regiochemical distribution of the acyl groups on the glycerol backbone [97]. The structure of MGDG and DGDG was fully elucidated by NMR, HPLC/ESI-ITMS and by ESI-ITMS after the enzymatic hydrolysis at $s n-1$ by lipase XI, which allowed the identification of the FA position. The dinoflagellates examined were divided into two clusters based on the forms of the MGDG and DGDG profiles [100]. One group showed MGDG (18:5/18:4), MGDG (18:5/18:5), DGDG (18:4/18:4) and DGDG (18:5/18:4) as major forms, and the second one showed major MGDG (20:5/18:4), MGDG (20:5/18:5), DGDG (20:5/18:4) and DGDG (20:5/18:5) forms. The differentiation between $\mathrm{C}_{18} / \mathrm{C}_{18}$ and $\mathrm{C}_{20} / \mathrm{C}_{18}$ glycolipids indicates the predominance of different biosynthetic pathways, as well as a possible evolutionary divergence between the two dinoflagellate clusters [100]. Furthermore, these unusual octacosapolyenoic FAs seem favorable for distinguishing possible evolutionary divergence between species [100]. This knowledge was never accessed before due to the limitations of traditional methodologies, such as GC/MS analysis, which did not allow the association of each fatty acid with the molecular species of glycolipids. Moreover, the distinguishable difference between the GL profile of warm- and cold-adapted was the level of unsaturation in the sn-2 fatty acid of DGDG, which was found to be almost exclusively 18:5n-3 in cold-adapted species and a mixture of 18:5n-3 and 18:4n-3 in warm-adapted species [43]. Leblond et al. (2010) observed in different species of the $C_{20} / C_{18}$ peridinin-containing dinoflagellate Pyrocystis sp. that fatty acid modulation occurred consistently within the sn-2 fatty acid of DGDG according to growth temperature [101]. The modulation occurred 
between the 18:5n-3 and 18:4n-3 forms at the sn-2 fatty acid of DGDG, while the sn-1 fatty acid 20:5n-3 remained constant. Therefore, maximum unsaturation of the $\mathrm{C}_{18} s n-2$ fatty acid of DGDG to produce $18: 5 n-3$ is a key response to temperature for these algae [43,101]. Also, trigalactosyl diacylglycerol (TGDG) lipids were newly described in dinoflagellates [43].

Due to the large variety of genera lineage of dinoflagellates, much information is still unknown about glycolipids' signature and biosynthetic pathways. Future studies are needed to determine the biosynthesis and function of TGDG in dinoflagellates and are also needed to examine the modifications on the lipidome of dinoflagellates under various growth conditions, such as temperature, with a particular interest in the species preferably producing 20:5 FA $[100,101]$.

\subsubsection{Glycolipidomic Profiling on Eustigmatophyta}

Regarding the Eustigmatophyta, lipidomics studies were mainly focused on Nannochloropsis sp. $[14,52,95,102]$, including Nannochloropsis oculata and NMBluh014 and NMBluh-X strains (Table 2). All lipidomic studies of Heterokontophyta reported the MGDG, DGDG and SQDG profile, but, depending on the papers, different molecular species where detected, which is maybe due to the variation of the lipidome within different strains of microalgae. Also, the different methodologies and mass analyzers may lead to distinct information of the lipidome. He et al. [14] profiled the glycolipids of Nannochloropsis oculata and unequivocally identified 46 molecular species of MGDG, MGMG, DGDG, SQMG and SQDG. The glycolipidome contained $\mathrm{C}_{14-20}$ and the most abundant molecular species were MGDG (20:5/20:5), DGDG (20:5/16:0) and (20:5/16:1). Nannochloropsis sp. [52] was mainly composed of the molecular species SQDG (16:1/16:0), MGDG (20:5/14:0), DGDG (20:5/16:0), DGDG (20:5/16:1) and DGDG (20:5/14:0), in agreement with the results obtained for Nannochloropsis oculata [14]. Moreover, Li et al. [102] profiled two Nannochloropsis oceanic strains, NMBluh014 and NMBluh- $X$, and have discovered 30 discriminant polar lipid biomarkers providing the lipidomic differences for chemotaxonomy. The molecular composition of glycolipids of the two strains was remarkably different regarding the MGDG and SQDG species. MGDG (18:3/16:3), MGDG (20:5/14:0), SQDG (18:1/16:0) and SQDG (18:3/16:0) were only detected in NMBluh-X. Also, SQDG biomarkers containing molecular species with $C_{16}$ FA and MGDG biomarkers containing $C_{16}$ and $C_{20}$ FAs were clearly detectable in NMBluh-X but there were only traces in NMBluh014. This last strain contains DGDG (18:3/16:2). The NMBluh014's glycolipidome is remarkably different from the remaining species of Nannochloropsis reported until now.

Moreover, by using the MS-based approach it was possible to distinguish the taxonomic variation of Heterokont's glycolipidome of Eustigmatophyta Nannochloropsis that biosynthesize glycolipids containing 20:5 FA, and the Labyrinthulomycetes Schizochytrium that contains trace amounts of GLs, but contains GLs bearing 22:6 fatty acyl species [52]. One of the promising applications of the MS-based strategy concerns the profiling of the lipidome under environmental stress manipulation and nutritional variations [10,95]. The response of Nannochloropsis sp. to $N$ deprivation [97] induced the decrease in the content of glycolipids, containing several MGDG and DGDG molecular species with abundant 20:5 FA, and 14:0 and 16:0/1 shorter FA. SQDG was the most abundant lipid in Nannochloropsis sp., mainly the SQDG (16:0/16:1). The MS-based approach allowed the identification of MGDG (18:3/16:3), MGDG (18:3/16:4), MGDG (18:1/16:3) and DGDG (18:2/16:0) species as important biomarkers under $\mathrm{N}$ deprivation. One key difference in the overall molecular profile of Nannochloropsis sp. is that it produces EPA (20:5), and has less effect on $\mathrm{N}$ deprivation within this strain.

\subsubsection{Glycolipidomic Profiling on Prymnesiophyta}

The Haptophyta/Prymnesiophyta I. galbana was analyzed by LC-MS/MS during distinct stages of the growth cycle [72]. All types of GLs (MGDG, DGDG and SQDG) in I. galbana were significantly increased from the exponential phase to the end of the stationary phase. This microalgae distinguishes from most of the other categories due to its ability to biosynthesize docosahexaenoic acids (DHAs). 
DHA were found in MGDG and SQDG lipids at the stationary stage of I. galbana and a higher content of DHA-rich lipids could be obtained at the end of the stationary phase.

\subsubsection{Glycolipidomic Profiling on Rhodophyta}

Regarding the red microalgae, only two studies were performed using MS-based approaches characterized to study the glycolipidome of Porphyridium sp. by the analysis of isolated fractions of total lipid extract. The extract, rich in SQDGs, was found to contain SQDG (18:2/16:0), SQDG (20:4/16:0) and SQDG (20:5/16:0) [19]. Meanwhile, the analysis of the glycolipids-rich extract of Porphyridium aerugineum allowed to identify five MGDG molecular species as MGDG (20:4/18:3), MGDG (20:4/16:0), MGDG (20:5/16:0), MGDG (20:5/18:3) and MGDG (20:5/20:4) [73]. In these particular cases, MS was successfully applied to identify the glycolipids on particular extracts used to evaluate the anti-proliferative and antioxidant activities $[19,57,73]$.

\section{Final Considerations and Perspectives}

Throughout the present review paper, information has been provided as regards the significance of microalgae as a valuable reservoir of glycolipids with promising bioactive properties such as anti-viral, anti-inflammatory and antitumor promoters. These lipids have high biotechnological potential for food and health applications, and thus their structural elucidation is mandatory for understanding their structure-biological activity relationships.

Mass spectrometry-based approaches have been presented as valuable analytical tools for identification and quantification of glycolipids in routine analysis of microalgae. By using MS-based approaches, more than 20 monogalactosyldiacyl glycerolipids, 20 digalactosyl diacylglycerolipids and 35 sulfoquinovosyl diacylglycerolipids molecular species were identified. This confirms the great advantage of MS-based lipidomics for the global identification of GLs from the extracts of marine microalgae, including GL classes, fatty acyl composition and the location of fatty acids (sn-1 vs. sn-2). MGDGs are usually the most abundant class of GLs, accounting for about $0.1 \%-70 \%$ of the total lipids. DGDGs and anionic SQDGs account for about $0.02 \%-50 \%$ and $0.1 \%-40 \%$ of the total lipids, respectively. The content and composition of GLs and their fatty acids are tightly related to the state of chloroplast membranes, the taxonomic variability, the environmental stress parameters (light, salinity, temperature, contaminants) and the nutrient availability conditions. GL signature has taxonomic significance and can be used to evaluate lipid metabolism and the responsive pathways of microalgae, so far underexploited.

Microalgae present a metabolic plasticity, meaning that they can be explored and modulated by changing the cultivation conditions or even by metabolic engineering approaches for the production of GL molecular species with high added value. High-value glycolipids from microalgae raise big expectations in the context of bioeconomy as a new generation of products with enhanced nutritional and functional qualities. Future challenges include establishing the full profile of GLs at the molecular level within intra and inter taxonomy, a better understanding of the metabolic pathways of synthesis and a comprehensive screening of the bioactive compounds produced by microalgae. The biotechnological process of customised production of biomass and bioactives recovery should be optimized, fostering their potential applications as functional ingredients or extracts in final marketable products. Only the cooperation of scientists in phycology, chemistry, biology, agronomy, food and engineering is able to address these major challenges.

Acknowledgments: Acknowledgements are due to the University of Aveiro, Fundação para a Ciência e a Tecnologia (FCT, Portugal), European Union, QREN, POPH, FEDER and COMPETE for funding the QOPNA research unit (project PEst-C/QUI/UI0062/2013, FCOMP-01-0124-FEDER-037296), to RNEM (REDE/1504/REM/2005) for the Portuguese Mass Spectrometry Network. Elisabete da Costa (SFRH/BD/52499/2014) is grateful to the FCT for the grant.

Conflicts of Interest: The authors declare no conflict of interest. 


\section{References}

1. Adl, S.M.; Simpson, A.G.; Lane, C.E.; Lukes, J.; Bass, D.; Bowser, S.S.; Brown, M.; Burki, F.; Dunthorn, M.V.; Heiss, A.; et al. The revised classification of eukaryotes. J. Eukaryot. Microbiol. 2012, 59, 429-514. [CrossRef] [PubMed]

2. Cavalier-Smith, T.; Chao, E.E. Phylogeny and megasystematics of phagotrophic heterokonts (kingdom Chromista). J. Mol. Evol. 2006, 62, 388-420. [CrossRef] [PubMed]

3. Guiry, M.D.; Guiry, G.M. AlgaeBase; World-Wide Electronic Publication: National University of Ireland, Galway, Ireland. Available online: http:/ /www.algaebase.org (accessed on 23 March 2016).

4. Bellou, S.; Baeshen, M.N.; Elazzazy, A.M.; Aggeli, D.; Sayegh, F.; Aggelis, G. Microalgal lipids biochemistry and biotechnological perspectives. Biotechnol. Adv. 2014, 32, 1476-1493. [CrossRef] [PubMed]

5. Vigani, M.; Parisi, C.; Rodríguez-Cerezo, E.; Barbosa, M.J.; Sijtsma, L.; Ploeg, M.; Enzing, C. Food and feed products from micro-algae: Market opportunities and challenges for the EU. Trends Food Sci. Technol. 2015, 42, 81-92. [CrossRef]

6. Chu, W. Biotechnological applications of microalgae. IeJSME 2012, 6, 24-37.

7. Mostafa, S.S.M. Microalgal biotechnology: Prospects and applications. In Plant Science; InTech: Rijeka, Croatia, 2012; pp. 275-313.

8. Pulz, O.; Gross, W. Valuable products from biotechnology of microalgae. Appl. Microbiol. Biotechnol. 2004, 65, 635-648. [CrossRef] [PubMed]

9. Ibañez, E.; Cifuentes, A. Benefits of using algae as natural sources of functional ingredients. J. Sci. Food Agric. 2013, 93, 703-709. [CrossRef] [PubMed]

10. Guschina, I.A.; Harwood, J.L. Lipids and lipid metabolism in eukaryotic algae. Prog. Lipid Res. 2006, 45, 160-186. [CrossRef] [PubMed]

11. Harwood, J.L.; Guschina, I.A. The versatility of algae and their lipid metabolism. Biochimie 2009, 91, 679-684. [CrossRef] [PubMed]

12. Siegenthaler, P.A.; Murata, N. Lipids in Photosynthesis: Structure, Function and Genetics; Kluwer Academic Publishers: Dordrecht, The Netherlands, 1998; pp. 21-81.

13. Hölzl, G.; Dörmann, P. Structure and function of glycoglycerolipids in plants and bacteria. Prog. Lipid Res. 2007, 46, 225-243. [CrossRef] [PubMed]

14. He, H.; Rodgers, R.P.; Marshall, A.G.; Hsu, C.S. Algae polar lipids characterized by online liquid chromatography coupled with hybrid linear quadrupole ion trap/fourier transform ion cyclotron resonance mass spectrometry. Energy Fuels 2011, 25, 4770-4775. [CrossRef]

15. Kim, S.-H.; Liu, K.-H.; Lee, S.-Y.; Hong, S.-J.; Cho, B.-K.; Lee, H.; Lee, C.-G.; Choi, H.-K. Effects of light intensity and nitrogen starvation on glycerolipid, glycerophospholipid, and carotenoid composition in Dunaliella tertiolecta culture. PLoS ONE 2013, 8, e72415. [CrossRef] [PubMed]

16. Plouguerné, E.; de Souza, L.M.; Sassaki, G.L.; Cavalcanti, J.F.; Villela Romanos, M.T.; da Gama, B.A.P.; Pereira, R.C.; Barreto-Bergter, E. Antiviral Sulfoquinovosyldiacylglycerols (SQDGs) from the Brazilian brown seaweed Sargassum vulgare. Mar. Drugs 2013, 11, 4628-4640. [CrossRef] [PubMed]

17. Plouguerné, E.; da Gama, B.A.P.; Pereira, R.C.; Barreto-Bergter, E. Glycolipids from seaweeds and their potential biotechnological applications. Front. Cell. Infect. Microbiol. 2014, 4, 174. [CrossRef] [PubMed]

18. Noda, H.; Amano, H.; Arashima, K.; Nisizawa, K. Antitumor activity of marine algae. Hydrobiologia 1990, 204, 577-584. [CrossRef]

19. Naumann, I.; Darsow, K.H.; Walter, C.; Lange, H.A.; Buchholz, R. Identification of sulfoglycolipids from the alga Porphyridium purpureum by matrix-assisted laser desorption/ionisation quadrupole ion trap time-of-flight mass spectrometry. Rapid Commun. Mass Spectrom. 2007, 21, 3185-3192. [CrossRef] [PubMed]

20. Reshef, V.; Mizrachi, E.; Maretzki, T.; Silberstein, C.; Loya, S.; Hizi, A.; Carmeli, S. New acylated sulfoglycolipids and digalactolipids and related known glycolipids from cyanobacteria with a potential to inhibit the reverse transcriptase of HIV-1. J. Nat. Prod. 1997, 60, 1251-1260. [CrossRef] [PubMed]

21. Morimoto, T. Anti-tumour-promoting glyceroglycolipids from the green alga, Chlorella vulgaris. Phytochemistry 1995, 40, 1433-1437. [CrossRef]

22. Zhang, J.; Li, C.; Yu, G.; Guan, H. Total synthesis and structure-activity relationship of glycoglycerolipids from marine organisms. Mar. Drugs 2014, 12, 3634-3659. [CrossRef] [PubMed] 
23. Stengel, D.B.; Connan, S.; Popper, Z.A. Algal chemodiversity and bioactivity: Sources of natural variability and implications for commercial application. Biotechnol. Adv. 2011, 29, 483-501. [CrossRef] [PubMed]

24. Yan, X.; Chen, D.; Xu, J.; Zhou, C. Profiles of photosynthetic glycerolipids in three strains of Skeletonema determined by UPLC-Q-TOF-MS. J. Appl. Phycol. 2011, 23, 271-282. [CrossRef]

25. Yan, X.; Li, H.; Xu, J.; Zhou, C. Analysis of phospholipids in microalga Nitzschia closterium by UPLC-Q-TOF-MS. Chin. J. Oceanol. Limnol. 2010, 28, 106-112. [CrossRef]

26. Vieler, A.; Wilhelm, C.; Goss, R.; Süß, R.; Schiller, J. The lipid composition of the unicellular green alga Chlamydomonas reinhardtii and the diatom Cyclotella meneghiniana investigated by MALDI-TOF MS and TLC. Chem. Phys. Lipids 2007, 150, 143-155. [CrossRef] [PubMed]

27. Xu, J.; Chen, D.; Yan, X.; Chen, J.; Zhou, C. Global characterization of the photosynthetic glycerolipids from a marine diatom Stephanodiscus sp. by ultra performance liquid chromatography coupled with electrospray ionization-quadrupole-time of flight mass spectrometry. Anal. Chim. Acta 2010, 663, 60-68. [CrossRef] [PubMed]

28. Li, H.; Yan, X.; Xu, J.; Zhou, C. Precise identification of photosynthetic glycerolipids in microalga Tetraselmis chuii by UPLC-ESI-Q-TOF-MS. Sci. China Ser. C Life Sci. 2008, 51, 1101-1107. [CrossRef] [PubMed]

29. Petroutsos, D.; Amiar, S.; Abida, H.; Dolch, L.-J.; Bastien, O.; Rébeillé, F.; Jouhet, J.; Falconet, D.; Block, M.A.; McFadden, G.I.; et al. Evolution of galactoglycerolipid biosynthetic pathways-From cyanobacteria to primary plastids and from primary to secondary plastids. Prog. Lipid Res. 2014, 54, 68-85. [CrossRef] [PubMed]

30. Mühlroth, A.; Li, K.; Røkke, G.; Winge, P.; Olsen, Y.; Hohmann-Marriott, M.F.; Vadstein, O.; Bones, A.M. Pathways of lipid metabolism in marine algae, co-expression network, bottlenecks and candidate genes for enhanced production of EPA and DHA in species of Chromista. Mar. Drugs 2013, 11, 4662-4697. [CrossRef] [PubMed]

31. Lee, A.G. Membrane lipids: It's only a phase. Curr. Biol. 2000, 10, 377-380. [CrossRef]

32. Lu, N.; Wei, D.; Chen, F.; Yang, S.-T. Lipidomic profiling and discovery of lipid biomarkers in snow alga Chlamydomonas nivalis under salt stress. Eur. J. Lipid Sci. Technol. 2012, 114, 253-265. [CrossRef]

33. Lu, N.; Wei, D.; Chen, F.; Yang, S.-T. Lipidomic profiling reveals lipid regulation in the snow alga Chlamydomonas nivalis in response to nitrate or phosphate deprivation. Process Biochem. 2013, 48, 605-613. [CrossRef]

34. Yang, D.; Song, D.; Kind, T.; Ma, Y.; Hoefkens, J.; Fiehn, O. Lipidomic analysis of Chlamydomonas reinhardtii under nitrogen and sulfur deprivation. PLoS ONE 2015, 10, e0137948. [CrossRef] [PubMed]

35. Wang, Z.; Benning, C. Chloroplast lipid synthesis and lipid trafficking through ER-plastid membrane contact sites. Biochem. Soc. Trans. 2012, 40, 457-463. [CrossRef] [PubMed]

36. Boudière, L.; Michaud, M.; Petroutsos, D.; Rébeillé, F.; Falconet, D.; Bastien, O.; Roy, S.; Finazzi, G.; Rolland, N.; Jouhet, J.; et al. Glycerolipids in photosynthesis: Composition, synthesis and trafficking. Biochim. Biophys. Acta Bioenerg. 2014, 1837, 470-480. [CrossRef] [PubMed]

37. Baba, M.; Shiraiwa, Y. Biosynthesis of lipids and hydrocarbons in algae. In Photosynthesis; Dubinsky, Z., Ed.; InTech: Rijeka, Croatia, 2013.

38. Benning, C. Biosynthesis and function of the sulfolipid sulfoquinovosyl diacylglicerol. Annu. Rev. Plant Physiol. 1998, 49, 53-75. [CrossRef] [PubMed]

39. Gustafson, K.R.; Cardellina, J.H.; Fuller, R.W.; Weislow, O.S.; Kiser, R.F.; Snader, K.M.; Patterson, G.M.; Boyd, M.R. AIDS-antiviral sulfolipids from cyanobacteria (blue-green algae). J. Natl. Cancer Inst. 1989, 81, 1254-1258. [CrossRef] [PubMed]

40. Zianni, R.; Bianco, G.; Lelario, F.; Losito, I.; Palmisano, F.; Cataldi, T.R.I. Fatty acid neutral losses observed in tandem mass spectrometry with collision-induced dissociation allows regiochemical assignment of sulfoquinovosyl-diacylglycerols. J. Mass Spectrom. 2013, 48, 205-215. [CrossRef] [PubMed]

41. Abrol, Y.P.; Ahmad, A. Sulphur in Plants; Kluwer Academic: Dordrecht, The Netherlands, 2003; pp. $189-219$.

42. Kim, Y.H.; Choi, J.; Yoo, J.S.; Park, Y.; Kim, M.S. Structural identification of glycerolipid molecular species isolated from Cyanobacterium Synechocystis sp. PCC 6803 using Fast Atom Bombardment Tandem Mass Spectrometry. Anal. Biochem. 1999, 267, 260-270. [CrossRef] [PubMed]

43. Gray, C.G.; Lasiter, A.D.; Leblond, J.D. Mono- and digalactosyldiacylglycerol composition of dinoflagellates. III. Four cold-adapted, peridinin-containing taxa and the presence of trigalactosyldiacylglycerol as an additional glycolipid. Eur. J. Phycol. 2009, 44, 441-447. [CrossRef] 
44. Li-Beisson, Y.; Shorrosh, B.; Beisson, F.; Andersson, M.; Arondel, V.; Bates, P.; Baud, S.; Bird, D.; DeBono, A.; Durrett, T.; et al. Acyl lipid metabolism. In The Arabidopsis Book; Keiko, T., Ed.; American Society of Plant Biologists: Rockville, MD, USA, 2013; Volume 8, pp. 1-65.

45. Browse, J.; Somerville, C. Glycerolipid metabolism: Biochemistry and regulation. Annu. Rev. Plant Physiol. Plant Mol. Biol. 1991, 42, 467-506. [CrossRef]

46. Browse, J.; Warwick, N.; Somerville, C.R.; Slack, C.R. Fluxes through the prokaryotic and eukaryotic pathways of lipid synthesis in the "16:3" plant Arabidopsis thaliana. Biochem. J. 1986, 235, 25-31. [CrossRef] [PubMed]

47. Dormann, P.; Benning, C. Galactolipids rule in seed plants. Trends Plant Sci. 2002, 7, 112-118. [CrossRef]

48. Okazaki, Y.; Kamide, Y.; Hirai, M.Y.; Saito, K. Plant lipidomics based on hydrophilic interaction chromatography coupled to ion trap time-of-flight mass spectrometry. Metabolomics 2013, 9, 121-131. [CrossRef] [PubMed]

49. Khozin-Goldberg, I.; Cohen, Z. Unraveling algal lipid metabolism: Recent advances in gene identification. Biochimie 2011, 93, 91-100. [CrossRef] [PubMed]

50. Khozin-Goldberg, I.; Didi-Cohen, S.; Shayakhmetova, I.; Cohen, Z. Biosynthesis of eicosapentaenoic acid (EPA) in the freshwater eustigmatophyte Monodus subterraneus (Eustigmatophyceae). J. Phycol. 2002, 38, 745-756. [CrossRef]

51. Millar, A.A.; Smith, M.A.; Kunst, L. All fatty acids are not equal: Discrimination in plant membrane lipids. Trends Plant Sci. 2000, 5, 95-101. [CrossRef]

52. Yao, L.; Gerde, J.A.; Lee, S.-L.; Wang, T.; Harrata, K.A. Microalgae lipid characterization. J. Agric. Food Chem. 2015, 63, 1773-1787. [CrossRef] [PubMed]

53. Sandra, K.; Sandra, P. Lipidomics from an analytical perspective. Curr. Opin. Chem. Biol. 2013, 17, 847-853. [CrossRef] [PubMed]

54. Mayer, A.M.S.; Rodríguez, A.D.; Berlinck, R.G.S.; Fusetani, N. Marine pharmacology in 2007-2008: Marine compounds with antibacterial, anticoagulant, antifungal, anti-inflammatory, antimalarial, antiprotozoal, antituberculosis, and antiviral activities; affecting the immune and nervous system, and other miscellaneous mechanisms of action. Comp. Biochem. Physiol. C Toxicol. Pharmacol. 2011, 153, 191-222. [PubMed]

55. Amaro, H.; Guedes, A.; Malcata, F. Antimicrobial activities of microalgae: An invited review. In Science against Microbial Pathogens: Communicating Current Research and Technological Advances; Formatex: Badajoz, Spain, 2011; pp. 1272-1280.

56. Hamed, I.; Özogul, F.; Özogul, Y.; Regenstein, J.M. Marine Bioactive compounds and their health benefits: A review. Compr. Rev. Food Sci. Food Saf. 2015, 14, 446-465. [CrossRef]

57. Bergé, J.P.; Debiton, E.; Dumay, J.; Durand, P.; Barthomeuf, C. In vitro anti-inflammatory and anti-proliferative activity of sulfolipids from the red alga Porphyridium cruentum. J. Agric. Food Chem. 2002, 50, 6227-6232. [CrossRef] [PubMed]

58. Banskota, A.H.; Gallant, P.; Stefanova, R.; Melanson, R.; O’Leary, S.J.B. Monogalactosyldiacylglycerols, potent nitric oxide inhibitors from the marine microalga Tetraselmis chui. Nat. Prod. Res. 2013, 27, 1084-1090. [CrossRef] [PubMed]

59. Banskota, A.H.; Stefanova, R.; Gallant, P.; McGinn, P.J. Mono- and digalactosyldiacylglycerols: Potent nitric oxide inhibitors from the marine microalga Nannochloropsis granulata. J. Appl. Phycol. 2013, 25, 349-357. [CrossRef]

60. Banskota, A.H.; Stefanova, R.; Sperker, S.; Lall, S.; Craigie, J.S.; Hafting, J.T. Lipids isolated from the cultivated red alga Chondrus crispus inhibit nitric oxide production. J. Appl. Phycol. 2014, 26, 1565-1571. [CrossRef]

61. Bruno, A.; Rossi, C.; Marcolongo, G.; di Lena, A.; Venzo, A.; Berrie, C.P.; Corda, D. Selective in vivo anti-inflammatory action of the galactolipid monogalactosyldiacylglycerol. Eur. J. Pharmacol. 2005, 524, 159-168. [CrossRef] [PubMed]

62. Chirasuwan, N.; Chaiklahan, R.; Kittakoop, P.; Chanasattru, W.; Ruengitchatchawalya, M.; Tanticharoen, M.; Bunnag, B. Anti HSV-1 activity of sulphoquinovosyl diacylglycerol isolated from Spirulina platensis. Nat. Sci. 2007, 41, 311-318.

63. Hossain, Z.; Kurihara, H.; Hosokawa, M.; Takahashi, K. Growth inhibition and induction of differentiation and apoptosis mediated by sodium butyrate in Caco-2 cells with algal glycolipids. In Vitro Cell. Dev. Biol. 2005, 41, 154-159. [CrossRef] [PubMed] 
64. Naumman, I. Sulfoquinovosyldiacylglyceride Antiviral Active Substanzen. Ph.D. Thesis, Fakultät der Universitt Erlangen-Nür, Erlangen, Germany, 2009.

65. Mattos, B.B.; Romanos, M.T.V.; de Souza, L.M.; Sassaki, G.; Barreto-Bergter, E. Glycolipids from macroalgae: Potential biomolecules for marine biotechnology? Rev. Bras. Farmacogn. 2011, 21, 244-247. [CrossRef]

66. Plaza, M.; Herrero, M.; Alejandro Cifuentes, A.; Ibáñez, E. Innovative natural functional ingredients from microalgae. J. Agric. Food Chem. 2009, 57, 7159-7170. [CrossRef] [PubMed]

67. Bourre, J.-M. Dietary Omega-3 fatty acids for women. Biomed. Pharmacother. 2007, 61, 105-112. [CrossRef] [PubMed]

68. Spolaore, P.; Joannis-Cassan, C.; Duran, E.; Isambert, A. Commercial applications of microalgae. J. Biosci. Bioeng. 2006, 101, 87-96. [CrossRef] [PubMed]

69. Bergé, J.P.; Barnathan, G. Fatty acids from lipids of marine organisms: Molecular biodiversity, roles as biomarkers, biologically active compounds, and economical aspects. Adv. Biochem. Eng. Biotechnol. 2005, 96, 49-125. [PubMed]

70. Simopoulos, A. The importance of the ratio of Omega-6/Omega-3 essential fatty acids. Biomed. Pharmacother. 2002, 56, 365-379. [CrossRef]

71. Kagan, M.L.; Levy, A.; Leikin-Frenkel, A. Comparative study of tissue deposition of omega-3 fatty acids from polar-lipid rich oil of the microalgae Nannochloropsis oculata with krill oil in rats. Food Funct. 2015, 6, 186-192. [CrossRef] [PubMed]

72. Li, S.; Xu, J.; Chen, J.J.; Chen, J.J.; Zhou, C.; Yan, X. The major lipid changes of some important diet microalgae during the entire growth phase. Aquaculture 2014, 428-429, 104-110. [CrossRef]

73. Banskota, A.H.; Stefanova, R.; Sperker, S.; Melanson, R.; Osborne, J.A.; O’Leary, S.J.B. Five new galactolipids from the freshwater microalga Porphyridium aerugineum and their nitric oxide inhibitory activity. J. Appl. Phycol. 2013, 25, 951-960. [CrossRef]

74. Robertson, R.; Guihéneuf, F.; Bahar, B.; Schmid, M.; Stengel, D.; Fitzgerald, G.; Ross, R.; Stanton, C. The anti-inflammatory effect of algae-derived lipid extracts on lipopolysaccharide (LPS)-stimulated human THP-1 Macrophages. Mar. Drugs 2015, 13, 5402-5424. [CrossRef] [PubMed]

75. Ivanova, P.; Milne, S.; Myers, D.; Brown, H. Lipidomics: A mass spectrometry based systems level analysis of cellular lipids. Curr. Opin. Chem. Biol. 2009, 13, 526-531. [CrossRef] [PubMed]

76. Bou Khalil, M.; Hou, W.; Zhou, H.; Elisma, F.; Swayne, L.A.; Blanchard, A.P.; Yao, Z.; Bennett, S.A.L.; Figeys, D. Lipidomics era: Accomplishments and challenges. Mass Spectrom. Rev. 2010, 29, 877-929. [CrossRef] [PubMed]

77. Kataoka, N.; Misaki, A. Glycolipids from Spirulina maxima: Structure and fatty acid composition. Agric. Biol. Chem. 1983, 47, 2349-2355. [CrossRef]

78. Han, Y.; Wen, Q.; Chen, Z.; Li, P. Review of methods used for microalgal lipid-content analysis. Energy Proc. 2011, 12, 9444-9950. [CrossRef]

79. Leblond, J.D.; Chapman, P.J. Lipid class distribution of highly unsaturated long chain fatty acids in marine dionflagellates. J. Phycol. 2000, 1108, 1103-1108. [CrossRef]

80. Benning, C.; Huang, Z.H.; Gage, D.A. Accumulation of a novel glycolipid and a betaine lipid in cells of Rhodobacter sphaeroides grown under phosphate limitation. Arch. Biochem. Biophys. 1995, 317, 103-111. [CrossRef] [PubMed]

81. Son, B.W.; Kim, J.C.; Lee, S.M.; Cho, J.C.; Choi, J.S.; Choi, H.D.; Song, J.C. New diacylgalactolipids from the marine Cyanophycean microalga Oscillatoria sp. Bull. Korean Chem. Soc. 2000, 21, 1138-1140.

82. Son, B.W.; Cho, Y.J.; Cho, J.S.; Lee, W.K.; Kim, D.S.; Choi, H.D.; Choi, J.S.; Jung, J.H.; Im, K.S.; Choi, W.C. New galactolipids from the marine bacillariophycean microalga Nitzschia sp. Nat. Prod. Lett. 2001, 15, 299-306. [CrossRef] [PubMed]

83. Zhu, C.; Hu, P.; Liang, Q.-L.; Wang, Y.-M.; Luo, G.-A. Recent advances in lipidomics. Fenxi Huaxue/Chin. J. Anal. Chem. 2009, 37, 1390-1396. [CrossRef]

84. Watson, A.D. Thematic review series: Systems biology approaches to metabolic and cardiovascular disorders. Lipidomics: A global approach to lipid analysis in biological systems. J. Lipid Res. 2006, 47, 2101-2111. [CrossRef] [PubMed]

85. Cajka, T.; Fiehn, O. Comprehensive analysis of lipids in biological systems by liquid chromatography-mass spectrometry. Trends Anal. Chem. 2014, 61, 192-206. [CrossRef] [PubMed] 
86. Bligh, E.G.; Dyer, W.J. A rappid method of total lipid extraction and purification. Can. J. Biochem. Physiol. 1959, 37, 911-917. [CrossRef] [PubMed]

87. Folch, J.; Lees, M.; Sloane Stanley, G.H. A simple method for the isolation and purification of total lipides from animal tissues. J. Biol. Chem. 1957, 226, 497-509. [PubMed]

88. Kumar, R.R.; Rao, P.H.; Arumugam, M. Lipid extraction methods from microalgae: A comprehensive review. Front. Energy Res. 2015, 2. [CrossRef]

89. Li, Y.; Naghdi, F.G.; Garg, S.; Adarme-Vega, T.C.; Thurecht, K.J.; Ghafor, W.A.; Tannock, S.; Schenk, P.M. A comparative study: The impact of different lipid extraction methods on current microalgal lipid research. Microb. Cell Fact. 2014, 13. [CrossRef] [PubMed]

90. Zhou, L.; le Grandois, J.; Marchioni, E.; Zhao, M.; Ennahar, S.; Bindler, F. Improvement of total lipid and glycerophospholipid recoveries from various food matrices using pressurized liquid extraction. J. Agric. Food Chem. 2010, 58, 9912-9917. [CrossRef] [PubMed]

91. Cho, S.-C.; Choi, W.-Y.; Oh, S.-H.; Lee, C.-G.; Seo, Y.-C.; Kim, J.-S.; Song, C.-H.; Kim, G.-V.; Lee, S.-Y.; Kang, D.-H.; et al. Enhancement of lipid extraction from marine microalga, Scenedesmus associated with high-pressure homogenization process. J. Biomed. Biotechnol. 2012, 2012. [CrossRef] [PubMed]

92. Jones, J.; Manning, S.; Montoya, M.; Keller, K.; Poenie, M. Extraction of algal lipids and their analysis by HPLC and Mass Spectrometry. J. Am. Oil Chem. Soc. 2012, 89, 1371-1381. [CrossRef]

93. Herrero, M.; Vicente, M.J.; Cifuentes, A.; Ibáñez, E. Characterization by high-performance liquid chromatography / electrospray ionization quadrupole time-of-flight mass spectrometry of the lipid fraction of Spirulina platensis pressurized ethanol extract. Rapid Commun. Mass Spectrom. 2007, 21, 1729-1738. [CrossRef] [PubMed]

94. Christie, W.W.; Han, X. Lipid Analysis-Isolation, Separation, Identification and Lipidomic Analysis, 4th ed.; Oily Press: Bridgwater, UK; Woodhead Publishing Ltd.: Cambridge, UK, 2010.

95. Martin, G.J.O.; Hill, D.R.A.; Olmstead, I.L.D.; Bergamin, A.; Shears, M.J.; Dias, D.A.; Kentish, S.E.; Scales, P.J.; Botté, C.Y.; Callahan, D.L. Lipid profile remodeling in response to nitrogen deprivation in the microalgae Chlorella sp. (Trebouxiophyceae) and Nannochloropsis sp. (Eustigmatophyceae). PLoS ONE 2014, 9, e103389. [CrossRef] [PubMed]

96. Oradu, S.A.; Cooks, R.G. Multistep mass spectrometry methodology for direct characterization of polar lipids in green microalgae using paper spray ionization. Anal. Chem. 2012, 84, 10576-10585. [CrossRef] [PubMed]

97. Guella, G.; Frassanito, R.; Mancini, I. A new solution for an old problem: The regiochemical distribution of the acyl chains in galactolipids can be established by electrospray ionization tandem mass spectrometry. Rapid Commun. Mass Spectrom. 2003, 17, 1982-1994. [CrossRef] [PubMed]

98. Dodson, V.J.; Dahmen, J.L.; Mouget, J.-L.; Leblond, J.D. Mono- and digalactosyldiacylglycerol composition of the marennine-producing diatom, Haslea ostrearia: Comparison to a selection of pennate and centric diatoms. Phycol. Res. 2013, 61, 199-207. [CrossRef]

99. Su, X.; Xu, J.; Yan, X.; Zhao, P.; Chen, J.; Zhou, C.; Zhao, F.; Li, S. Lipidomic changes during different growth stages of Nitzschia closterium f. minutissima. Metabolomics 2013, 9, 300-310. [CrossRef]

100. Gray, C.G.; Lasiter, A.D.; Leblond, J.D. Mono- and digalactosyldiacylglycerol composition of dinoflagellates. I. Peridinin-containing taxa. Eur. J. Phycol. 2009, 44, 191-197. [CrossRef]

101. Leblond, J.D.; Dahmen, J.L.; Evens, T.J. Mono- and digalactosyldiacylglycerol composition of dinoflagellates. IV. Temperature-induced modulation of fatty acid regiochemistry as observed by electrospray ionization/mass spectrometry. Eur. J. Phycol. 2010, 45, 13-18. [CrossRef]

102. Li, S.; Xu, J.; Jiang, Y.; Zhou, C.; Yu, X.; Zhong, Y.; Chen, J.; Yan, X. Lipidomic analysis can distinguish between two morphologically similar strains of Nannochloropsis oceanica. J. Phycol. 2015, 51, 264-276. [CrossRef] [PubMed]

103. Hummel, J.; Segu, S.; Li, Y.; Irgang, S.; Jueppner, J.; Giavalisco, P. Ultra performance liquid chromatography and high resolution mass spectrometry for the analysis of plant lipids. Front. Plant Sci. 2011, 2, 1-17. [CrossRef] [PubMed]

104. Wuhrer, M.; de Boer, A.R.; Deelder, A.M. Structural glycomics using hydrophilic interaction chromatography (HILIC) with mass spectrometry. Mass Spectrom. Rev. 2009, 28, 192-206. [CrossRef] [PubMed]

105. Kind, T.; Okazaki, Y.; Saito, K.; Fiehn, O. LipidBlast templates as flexible tools for creating new in-silico tandem mass spectral libraries. Anal. Chem. 2014, 86, 11024-11027. [CrossRef] [PubMed] 
106. Kind, T.; Liu, K.H.; Lee, Y.; DeFelice, B.; Meissen, J.K.; Fiehn, O. LipidBlast in silico tandem mass spectrometry database for lipid identification. Nat. Methods. 2013, 10, 755-758. [CrossRef] [PubMed]

107. Costello, C.E.; Vath, J.E. Tandem mass spectrometry of glycolipids. Methods Enzymol. 1990, 193, 738-768. [PubMed]

108. Melo, T.; Alves, E.; Azevedo, V.; Martins, A.S.; Neves, B.; Domingues, P.; Calado, R.; Abreu, M.H.; Domingues, M.R. Lipidomics as a new approach for the bioprospecting of marine macroalgae: Unraveling the polar lipid and fatty acid composition of Chondrus crispus. Algal Res. 2015, 8, 181-191. [CrossRef]

109. Da Costa, E.; Melo, T.; Alves, E.; Domingues, P.; Calado, R.; Abreu, M.H.; Domingues, M.R. Decoding bioactive polar lipid profile of the macroalgae Codium tomentosum from a sustainable IMTA system using a lipidomic approach. Algal Res. 2015, 12, 388-397. [CrossRef]

110. Tatituri, R.V.V.; Brenner, M.B.; Turk, J.; Hsu, F.-F. Structural elucidation of diglycosyl diacylglycerol and monoglycosyl diacylglycerol from Streptococcus pneumoniae by Multiple-Stage Linear Ion-Trap Mass Spectrometry with Electrospray Ionization. J. Mass Spectrom. 2013, 47, 115-123. [CrossRef] [PubMed]

111. Navas-Iglesias, N.; Carrasco-Pancorbo, A.; Cuadros-Rodríguez, L. From lipids analysis towards lipidomics, a new challenge for the analytical chemistry of the 21st century. Part II: Analytical lipidomics. TrAC Trends Anal. Chem. 2009, 28, 393-403. [CrossRef]

112. Okazaki, Y.; Otsuki, H.; Narisawa, T.; Kobayashi, M.; Sawai, S.; Kamide, Y.; Kusano, M.; Aoki, T.; Hirai, M.Y.; Saito, K. A new class of plant lipid is essential for protection against phosphorus depletion. Nat. Commun. 2013, 4. [CrossRef] [PubMed]

113. Picchioni, G.A.; Watada, A.E.; Whitaker, B.D. Quantitative high-performance liquid chromatography analysis of plant phospholipids and glycolipids using light-scattering detection. Lipids 1996, 31, 217-221. [CrossRef] [PubMed]

114. Jamali, A.A.; Akbari, F.; Ghorakhlu, M.M.; de la Guardia, M.; Khosroushahi, A.Y. Applications of diatoms as potential microalgae in nanobiotechnology. BioImpacts 2012, 2, 83-89. [PubMed]

(C) 2016 by the authors; licensee MDPI, Basel, Switzerland. This article is an open access article distributed under the terms and conditions of the Creative Commons Attribution (CC-BY) license (http://creativecommons.org/licenses/by/4.0/). 